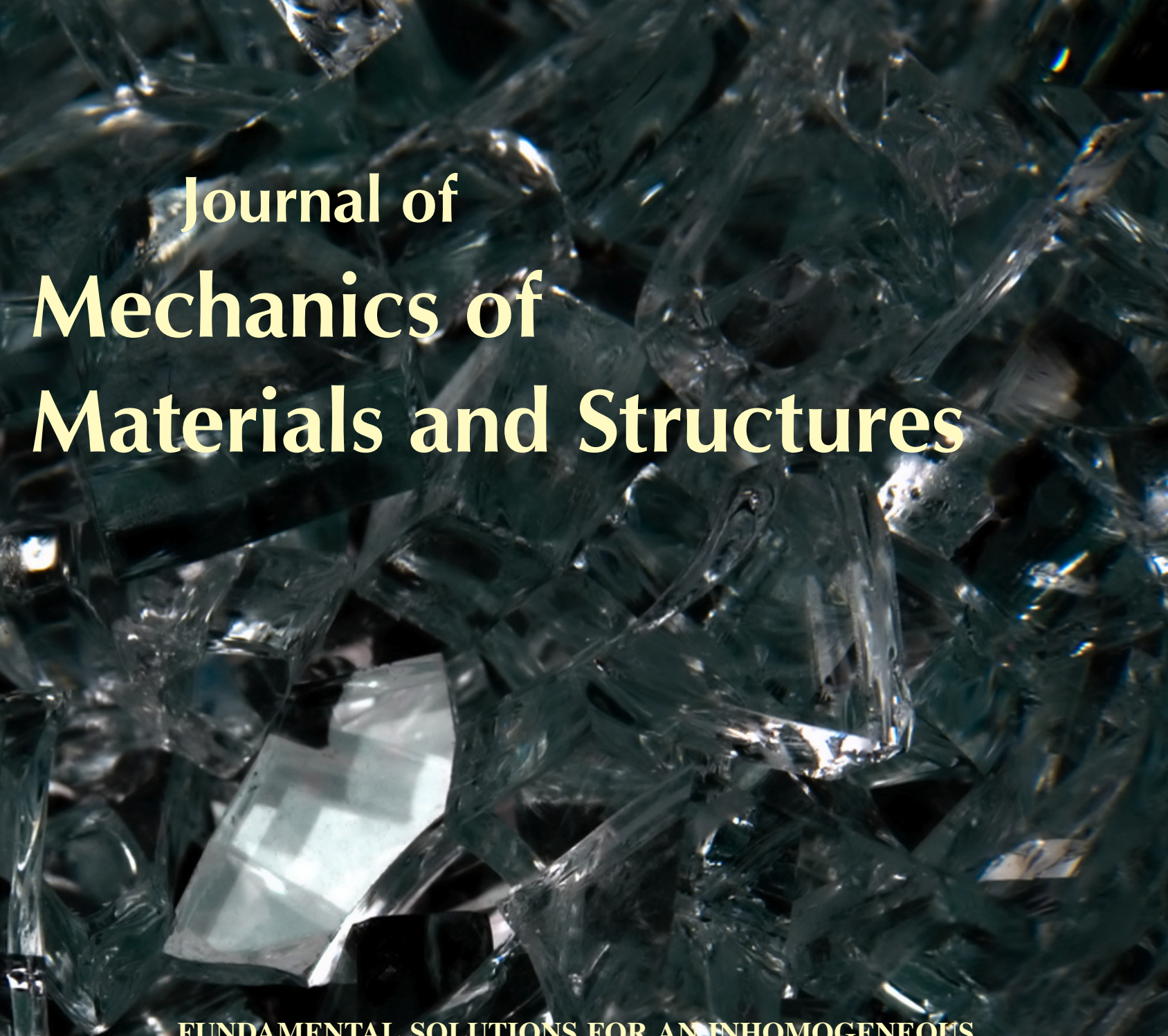

- F FUNDAMENTAL SOLUTIONS FOR ANHNHOMOGENEOÜS CROSS-ANISOTROPIC MATERIAL DUE TO HORIZONTAL AND VERTICAL If. $\%$ PLANE STRAIN LINE LOADS

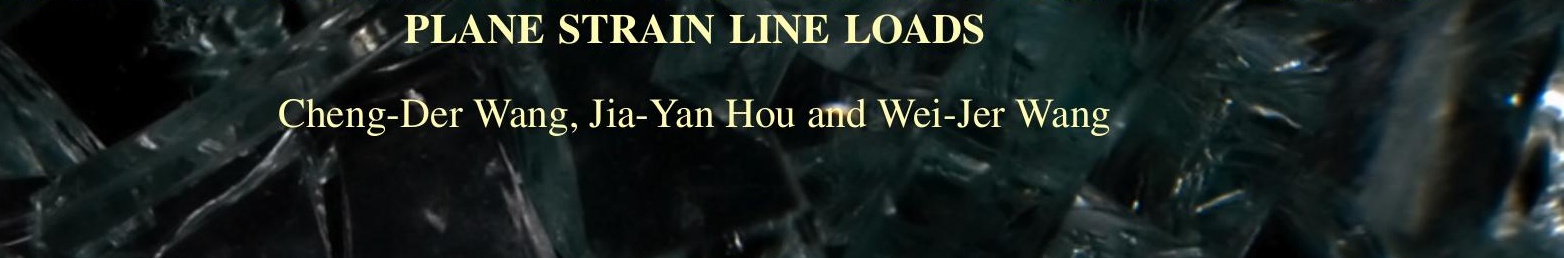

2

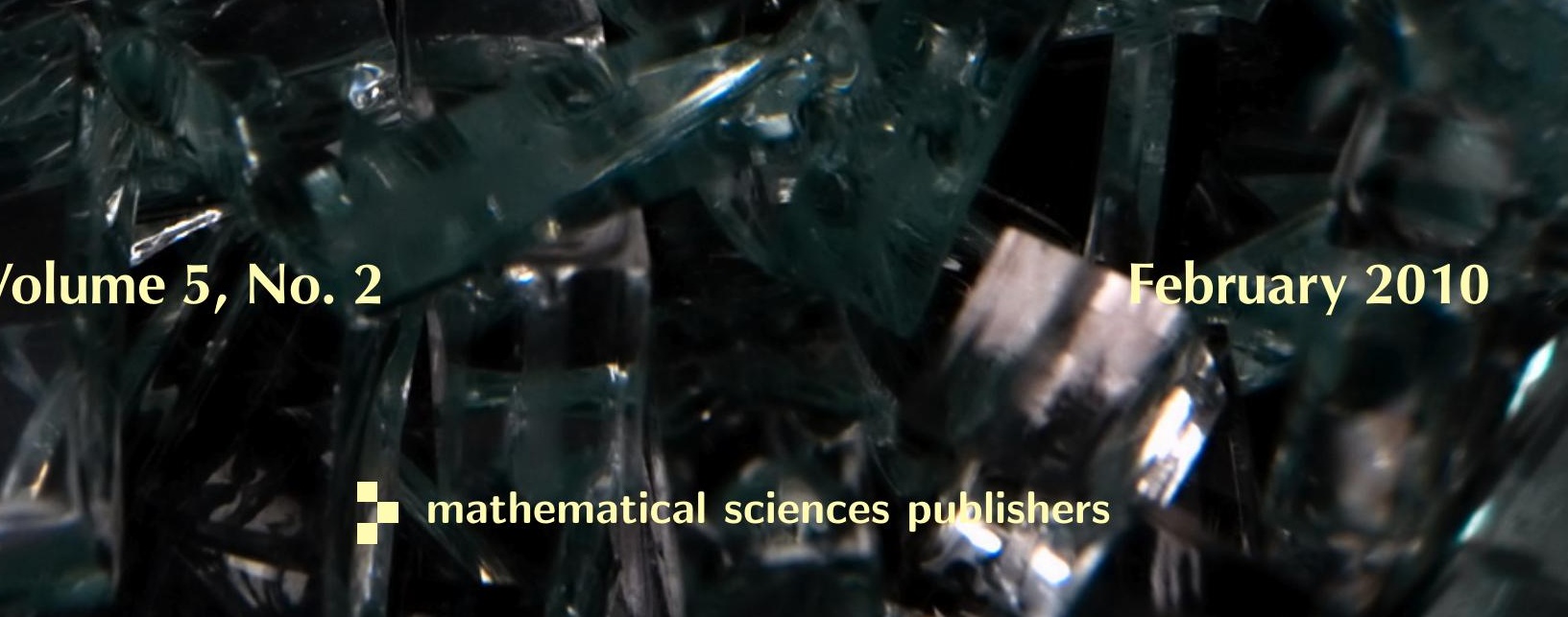




\title{
FUNDAMENTAL SOLUTIONS FOR AN INHOMOGENEOUS CROSS-ANISOTROPIC MATERIAL DUE TO HORIZONTAL AND VERTICAL PLANE STRAIN LINE LOADS
}

\author{
Cheng-Der WAng, JiA-YAn Hou AND WeI-Jer WANG
}

\begin{abstract}
This work derives the fundamental solutions for displacements and stresses due to horizontal and vertical line loads acting in a continuously inhomogeneous plane strain cross-anisotropic full space with Young's and shear moduli varying exponentially with depth. The governing equations can be obtained by combining the generalized Hooke's law, the strain-displacement relationships, and the equilibrium equations. Then, utilizing Fourier transforms, the governing equations are transformed into ordinary differential equations. Additionally, by using the variation of parameters, the solutions of the displacements in the Fourier domain are found. However, the stress solutions in the same domain can also be found by employing the stress-strain-displacement relationships. Eventually, performing inverse Fourier transforms by means of the numerical integration program QDAGI, the displacements and stresses induced by horizontal and vertical plane strain line loads can be calculated. The solutions indicate that the displacements and stresses are profoundly influenced by the nondimensional inhomogeneity parameter, the type and degree of material anisotropy, the types of loading, and the nondimensional horizontal distance. The proposed solutions are identical to those of Wang and Liao after suitable integration, as derived in an appendix, when the full space is a homogeneous cross-anisotropic material. A series of parametric studies are conducted to demonstrate the present solutions, and to elucidate the effects of aforementioned factors on the vertical normal stress. The results reveal that estimates of displacement and stress should take the inhomogeneity into account when studying cross-anisotropic materials under applied line loads.
\end{abstract}

\section{Introduction}

Many natural soils, such as flocculated clays, varved silts, or sands, are often deposited through a geologic process of sedimentation over a long period of time. The effects of deposition, overburden, desiccation, et cetera, can cause both anisotropic and inhomogeneous deformability. The mechanical responses of anisotropic materials with spatial gradients in composition, called anisotropic functionally graded materials, are important in many fields of applied mechanics. Hence, an elastic loading problem for a continuously inhomogeneous plane strain cross-anisotropic full space with Young's and shear moduli varying exponentially with depth is considered in this article.

The solution of a point load acting in the interior of a full space is called the fundamental solution or the Green's function solution. This kind of solution is employed in the boundary integral equation method (or boundary element method) for solving elastostatic boundary value problems [Hu et al. 2007]. However,

Keywords: inhomogeneity, cross-anisotropic full space, horizontal and vertical plane strain line loads, Fourier transforms, fundamental solutions of displacements and stresses, numerical integrations.

We acknowledge the financial support of the National Science Council (Taiwan) under Grant No. 96-2628-E-239-022-MY3. 
theoretical treatment of both inhomogeneous and anisotropic characteristics in materials frequently poses mathematical difficulties. Therefore, a plane strain state on the $y$-axis of a cartesian coordinate system is assumed throughout this study, which means the inhomogeneous cross-anisotropic medium is subjected to two-dimensional horizontal/vertical line loads, $P_{x} / P_{z}$ (force/length). Furthermore, the magnitudes and distributions of displacements and stresses in materials due to these applied loads have always played an important role in the design of foundations. In the past, a great deal of study was contributed to estimate the displacements and stresses in isotropic media with Young's or shear modulus varying with depth according to the power law, the linear law, the exponential law, et cetera. A very detailed survey of the solutions related to soil/rock mechanics and foundation engineering for inhomogeneous isotropic/crossanisotropic media is given in [Wang et al. 2003]. However, some recent investigations are not included in that survey, and will be mentioned here. Some relevant articles about inhomogeneous isotropic media are [Chen et al. 2001; Gray et al. 2001; Doherty and Deeks 2002; 2003c; 2003a; 2003b; 2005; 2006; Martin 2004; Martin et al. 2002; Cihan 2003], and some about inhomogeneous anisotropic media are [Azis and Clements 2001; Wang et al. 2003; 2006; Wang and Tzeng 2009]. Azis and Clements [2001] studied the solutions of boundary value problems for static deformations of inhomogeneous anisotropic elastic materials. The elastic moduli varied continuously with three cartesian coordinates, and three plane strain boundary value problems for inhomogeneous isotropic/cross-anisotropic media were illustrated. Wang et al. [2003] presented semianalytical solutions for displacements and stresses by employing the Hankel integral transforms in a cylindrical coordinate system with the assumption of axial symmetry about the $z$-axis, owing to a vertical point load in a continuously inhomogeneous cross-anisotropic half space with Young's and shear moduli varying exponentially with depth $\left(E e^{-k z}, E^{\prime} e^{-k z}\right.$, and $\left.G^{\prime} e^{-k z}\right)$. The planes of cross-anisotropy were horizontal, and the resulting integrals involved products of Bessel functions of the first kind, an exponential, a polynomial; some of them cannot be given in a closed form. Hence, numerical techniques proposed by Longman [1956b; 1956a] and Davis and Rabinowitz [1984] were adopted. The point load solutions in the Hankel domain for the half space were obtained by superposing the solutions of two full spaces, one with a point load in its interior and the other with opposite traction of the first full space along the boundary surface plane. Subsequently, Wang et al. [2006] integrated the point load solutions of [Wang et al. 2003] to get solutions for the displacements and stresses along the centerline, induced by a uniform vertical circular load acting in the interior of an inhomogeneous crossanisotropic half space with Young's and shear moduli varying exponentially with depth. In addition, Wang and Tzeng [2009] generated the solutions for the same medium for conical and parabolic vertical circular loads.

In this article, the fundamental solutions of displacements and stresses subjected to horizontal and vertical line loads for an inhomogeneous plane strain cross-anisotropic medium with Young's and shear moduli varying exponentially with depth $\left(E e^{-k z}, E^{\prime} e^{-k z}\right.$, and $\left.G^{\prime} e^{-k z}\right)$ are derived. Note that, in order to consider the important effect of a horizontal force on the inhomogeneous cross-anisotropic medium, the axisymmetric assumption cannot be utilized. In other words, the solutions of Wang et al. [2003] cannot be applied to solve for the displacements and stresses resulting from the actions of horizontal forces, or those from asymmetrically shaped loads, such as strip loads. Hence we use cartesian coordinates for performing Fourier transforms. However, past investigations suggest that considering anisotropic deformability together with inhomogeneity can make the problems too complicated to allow closed-form solutions for the displacement and stress; therefore, we resort to numerical integration using QDAGI. 
The present solutions indicate that the displacements and stresses are governed by the nondimensional inhomogeneity parameter $(k z)$, the type and degree of material anisotropy $\left(E / E^{\prime}, G^{\prime} / E^{\prime}\right.$, and $\left.v / v^{\prime}\right)$, the types of line loading $\left(P_{x}\right.$ and $P_{z}$, force/length), and the nondimensional horizontal distance $(x / z)$. The proposed solutions are identical with those of [Wang and Liao 1999] by integration, as derived in the Appendix of this article, when the full space is a homogeneous cross-anisotropic medium owing to infinite horizontal and vertical line loads. Finally, a parametric study is conducted to demonstrate the obtained solutions and to elucidate the effect of aforementioned factors on the vertical normal stress. The results reveal that the inhomogeneous characteristics should be taken into account in the case of cross-anisotropic soil/rock masses under plane strain horizontal/vertical line loads.

\section{Governing equations for an inhomogeneous plane strain cross-anisotropic medium due to line loads}

In this study, solutions for the displacements and stresses caused by horizontal/vertical line loads in a continuously inhomogeneous plane strain cross-anisotropic full space are derived. Figure 1 depicts a cartesian coordinate system in which the $z$-axis is the axis of rotation associated with elastic symmetry, and the $x-y$ plane is the cross-anisotropic plane. The cross-anisotropic medium has inhomogeneous elastic properties according with the assumptions of [Wang et al. 2003]. The Young's and shear moduli vary exponentially with depth, but the two Poisson's ratios remain constants. Hence, the expression of stress-strain for an inhomogeneous cross-anisotropic medium under the plane strain assumption is

$$
\left[\begin{array}{c}
\sigma_{x x} \\
\sigma_{z z} \\
\tau_{x z}
\end{array}\right]=\left[\begin{array}{ccc}
C_{11} & C_{13} & 0 \\
C_{13} & C_{33} & 0 \\
0 & 0 & C_{44}
\end{array}\right]\left[\begin{array}{c}
\varepsilon_{x x} \\
\varepsilon_{z z} \\
\gamma_{x z}
\end{array}\right] e^{-k z},
$$

where $k$ is referred to as the inhomogeneity parameter, and it has dimensions of inverse length. The product $\mathrm{kz}$ represents the nondimensional inhomogeneity parameter; here $z$ is coordinate (increasing

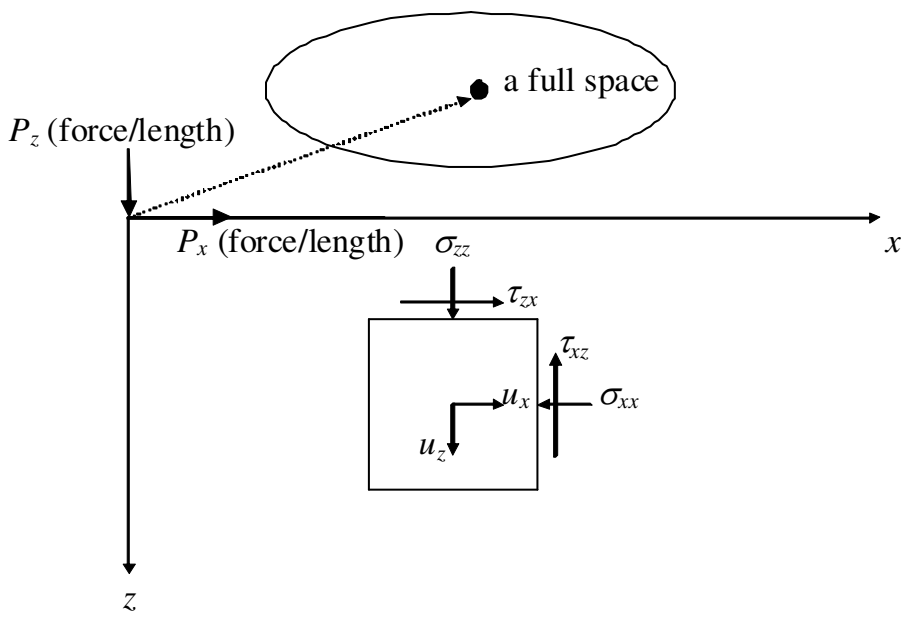

Figure 1. Horizontal/vertical line load $P_{x} / P_{z}$ acting in an inhomogeneous plane strain cross-anisotropic full space. 
downward) in the direction of the axis of rotation associated with elastic symmetry. The matrix entries $C_{11}, C_{13}, C_{33}$, and $C_{44}$ are elastic moduli or elasticity constants of the medium, and can be expressed in terms of five independent elastic constants $E, E^{\prime}, v, v^{\prime}$, and $G^{\prime}$ as

$$
C_{11}=\frac{E\left(1-\frac{E}{E^{\prime}} v^{\prime 2}\right)}{(1+v)\left(1-v-\frac{2 E}{E^{\prime}} v^{\prime 2}\right)}, \quad C_{13}=\frac{E v^{\prime}}{1-v-\frac{2 E}{E^{\prime}} v^{\prime 2}}, \quad C_{33}=\frac{E^{\prime}(1-v)}{1-v-\frac{2 E}{E^{\prime}} v^{\prime 2}}, \quad C_{44}=G^{\prime},
$$

where

- $E$ and $E^{\prime}$ are Young's moduli in the plane of cross-anisotropy and in the direction normal to it, respectively;

- $v$ and $v^{\prime}$ are Poisson's ratios characterizing the lateral strain response in the plane of cross-anisotropy to a stress acting parallel or normal to it, respectively; and

- $G^{\prime}$ is the shear modulus in planes normal to the plane of cross-anisotropy.

Since it is often convenient in soil mechanics to consider compressive stresses as positive, this convention is adopted in this article. The normal and shear stresses act in an element as shown in Figure 1, and the stresses are all of positive sign [Poulos and Davis 1974]. The differences between the homogeneous cross-anisotropic elastic constants [Wang and Liao 1999] and the inhomogeneous ones [Wang et al. 2003] in this work are listed in Table 1. For an inhomogeneous cross-anisotropic material as described by (1), only three engineering elastic constants $\left(E, E^{\prime}\right.$, and $\left.G^{\prime}\right)$ are exponentially dependent on the nondimensional inhomogeneity parameter $k z$; however, two Poisson's ratios $\left(v\right.$ and $\left.v^{\prime}\right)$ remain constants. In addition, according to the nondimensional inhomogeneity parameter $k$, three distinct situations exist:

- $k z>0$ indicates a hardened surface, where $E, E^{\prime}$, and $G^{\prime}$ decrease with increasing depth (for example, graded ceramic coatings on metallic substrates [Giannakopoulos and Suresh 1997]).

- $k z=0$ indicates the conventional homogeneous condition.

- $k z<0$ indicates a soft surface, where $E, E^{\prime}$, and $G^{\prime}$ increase with increasing of depth: for example, modulus variations measured as a function of depth beneath the earth's surface for soils and rocks [Giannakopoulos and Suresh 1997].

\begin{tabular}{|cc|}
\hline Homogeneous & Inhomogeneous \\
$E$ & $E e^{-k z}$ \\
$E^{\prime}$ & $E^{\prime} e^{-k z}$ \\
$v$ & $v$ \\
$v^{\prime}$ & $v^{\prime}$ \\
$G^{\prime}$ & $G^{\prime} e^{-k z}$ \\
\hline
\end{tabular}

Table 1. Differences between homogeneous [Wang and Liao 1999] and inhomogeneous [Wang et al. 2003] cross-anisotropic elastic constants. 
The expressions of the strain-displacement relations for small strain in a cartesian coordinate system are [Poulos and Davis 1974]:

$$
\varepsilon_{x x}=-\frac{\partial u_{x}}{\partial x}, \quad \varepsilon_{z z}=-\frac{\partial u_{z}}{\partial z}, \quad \gamma_{x z}=-\frac{\partial u_{x}}{\partial z}-\frac{\partial u_{z}}{\partial x},
$$

where $u_{x}$ and $u_{z}$ are the displacements in the $x$ and $z$ directions, as shown in Figure 1. A positive normal strain corresponds with a decrease in length, whereas a positive shear strain represents an increase in the right angle, and a negative shear strain denotes a decrease in the right angle [Poulos and Davis 1974].

By considering the equilibrium of the element shown in Figure 1 in a cartesian coordinate system, the following equilibrium equations are obtained [Poulos and Davis 1974]:

$$
\frac{\partial \sigma_{x x}}{\partial x}+\frac{\partial \tau_{x z}}{\partial z}-P_{x} \delta(x) \delta(z)=0, \quad \frac{\partial \tau_{x z}}{\partial x}+\frac{\partial \sigma_{z z}}{\partial z}-P_{z} \delta(x) \delta(z)=0,
$$

where $\delta$ is the Dirac delta function, and $P_{x}$ and $P_{z}$ (force/length) are the horizontal and vertical infinite line loads acting at the origin in an inhomogeneous cross-anisotropic full space.

Combining (1) and (3), and substituting them into (4), the Navier-Cauchy equations for an inhomogeneous plane strain cross-anisotropic medium can be regrouped as

$$
\begin{aligned}
& \left(C_{11} \frac{\partial^{2}}{\partial x^{2}}+C_{44} \frac{\partial^{2}}{\partial z^{2}}-k C_{44} \frac{\partial}{\partial z}\right) u_{x}+\left(\left(C_{13}+C_{44}\right) \frac{\partial^{2}}{\partial x \partial z}-k C_{44} \frac{\partial}{\partial x}\right) u_{z}+P_{x} \delta(x) \delta(z)=0 \\
& \left(\left(C_{13}+C_{44}\right) \frac{\partial^{2}}{\partial x \partial z}-k C_{13} \frac{\partial}{\partial x}\right) u_{x}+\left(C_{44} \frac{\partial^{2}}{\partial x^{2}}+C_{33} \frac{\partial^{2}}{\partial z^{2}}-k C_{33} \frac{\partial}{\partial z}\right) u_{z}+P_{z} \delta(x) \delta(z)=0 .
\end{aligned}
$$

\section{Fundamental solutions of displacements in the Fourier domain}

In order to solve the governing equations as expressed in (5), the Fourier transforms relating to the $x$ coordinate of the components of displacement are

$$
\frac{1}{\sqrt{2 \pi}} \int_{-\infty}^{\infty}\left\{\begin{array}{l}
u_{x}(x, z) \\
u_{z}(x, z)
\end{array}\right\} e^{-i \alpha x} d x=\left\{\begin{array}{l}
\bar{u}_{x}(\alpha, z) \\
\bar{u}_{z}(\alpha, z)
\end{array}\right\}
$$

where $\alpha$ is the Fourier transformed parameter. However, the Dirac delta function has the property $\int_{-\infty}^{\infty} \delta(x) d x=1$. Hence, (5) becomes a system of
ordinary differential equations:

$$
\begin{aligned}
& \left(-\alpha^{2} C_{11}+C_{44} \frac{d^{2}}{d z^{2}}-k C_{44} \frac{d}{d z}\right) \bar{u}_{x}+\left(i \alpha\left(C_{13}+C_{44}\right) \frac{d}{d z}-i \alpha k C_{44}\right) \bar{u}_{z}=-\frac{P_{x}}{\sqrt{2 \pi}} \delta(z), \\
& \left(i \alpha\left(C_{13}+C_{44}\right) \frac{d}{d z}-i \alpha k C_{13}\right) \bar{u}_{x}+\left(-\alpha^{2} C_{44}+C_{33} \frac{d^{2}}{d z^{2}}-k C_{33} \frac{d}{d z}\right) \bar{u}_{z}=-\frac{P_{z}}{\sqrt{2 \pi}} \delta(z) .
\end{aligned}
$$

The homogeneous solutions of (7) are found by solving the ordinary differential equations

$$
\begin{aligned}
& \bar{u}_{x}(H)=A_{1} e^{\left(k+u_{1}\right) z / 2}+A_{2} e^{\left(k-u_{1}\right) z / 2}+A_{3} e^{\left(k+u_{2}\right) z / 2}+A_{4} e^{\left(k-u_{2}\right) z / 2}, \\
& \bar{u}_{z}(H)=B_{1} e^{\left(k+u_{1}\right) z / 2}+B_{2} e^{\left(k-u_{1}\right) z / 2}+B_{3} e^{\left(k+u_{2}\right) z / 2}+B_{4} e^{\left(k-u_{2}\right) z / 2},
\end{aligned}
$$


where

$$
u_{1}, u_{2}=\sqrt{\frac{2 \alpha^{2} t+k^{2} C_{33} C_{44} \mp 2 \alpha \sqrt{\alpha^{2} t^{2}-4\left(\alpha^{2} C_{11}+k^{2} C_{13}\right) C_{33} C_{44}^{2}}}{C_{33} C_{44}}},
$$

with $t=C_{11} C_{33}-C_{13}^{2}-2 C_{13} C_{44}$. (The top sign is used for $u_{1}$.)

The coefficients $B_{i}$ can be eliminated in favor of the $A_{i}$ by substituting (8) into (7). This reduces equations (8) to

$$
\begin{aligned}
& \bar{u}_{x}(H)=A_{1} e^{\left(k+u_{1}\right) z / 2}+A_{2} e^{\left(k-u_{1}\right) z / 2}+A_{3} e^{\left(k+u_{2}\right) z / 2}+A_{4} e^{\left(k-u_{2}\right) z / 2}, \\
& \bar{u}_{z}(H)=S_{1} A_{1} e^{\left(k+u_{1}\right) z / 2}+S_{2} A_{2} e^{\left(k-u_{1}\right) z / 2}+S_{3} A_{3} e^{\left(k+u_{2}\right) z / 2}+S_{4} A_{4} e^{\left(k-u_{2}\right) z / 2},
\end{aligned}
$$

where

$$
\begin{array}{ll}
S_{1}=\frac{-2 i \alpha\left(C_{13}\left(k-u_{1}\right)-C_{44}\left(k+u_{1}\right)\right)}{4 \alpha^{2} C_{44}+C_{33}\left(k^{2}-u_{1}^{2}\right)}, & S_{2}=\frac{-2 i \alpha\left(C_{13}\left(k+u_{1}\right)-C_{44}\left(k-u_{1}\right)\right)}{4 \alpha^{2} C_{44}+C_{33}\left(k^{2}-u_{1}^{2}\right)}, \\
S_{3}=\frac{-2 i \alpha\left(C_{13}\left(k-u_{2}\right)-C_{44}\left(k+u_{2}\right)\right)}{4 \alpha^{2} C_{44}+C_{33}\left(k^{2}-u_{2}^{2}\right)}, & S_{4}=\frac{-2 i \alpha\left(C_{13}\left(k+u_{2}\right)-C_{44}\left(k-u_{2}\right)\right)}{4 \alpha^{2} C_{44}+C_{33}\left(k^{2}-u_{2}^{2}\right)} .
\end{array}
$$

Next, the particular solutions of (7) can be found by defining two displacement functions:

- For $z \geq 0$ (recall that the sign of $z$ is downward positive):

$$
\begin{aligned}
& \bar{u}_{x}(P)=C_{1} e^{\left(k+u_{1}\right) z / 2}+C_{2} e^{\left(k-u_{1}\right) z / 2}+C_{3} e^{\left(k+u_{2}\right) z / 2}+C_{4} e^{\left(k-u_{2}\right) z / 2}, \\
& \bar{u}_{z}(P)=D_{1} e^{\left(k+u_{1}\right) z / 2}+D_{2} e^{\left(k-u_{1}\right) z / 2}+D_{3} e^{\left(k+u_{2}\right) z / 2}+D_{4} e^{\left(k-u_{2}\right) z / 2} .
\end{aligned}
$$

- For $z<0: \bar{u}_{x}(P)=0, \bar{u}_{z}(P)=0$.

The undetermined coefficients $C_{i}$ and $D_{i}(i=1, \ldots, 4)$ can be obtained by variation of parameters:

$$
\begin{aligned}
C_{1}, C_{2}= & \frac{-4\left(\left(\frac{1}{4} C_{33}\left(k \pm u_{1}\right)^{2}-\frac{1}{2} C_{33} k\left(k \pm u_{1}\right)-\alpha^{2} C_{44}\right) P_{x}-i \alpha\left(\frac{1}{2}\left(C_{13}+C_{44}\right)\left(k \pm u_{1}\right)-k C_{44}\right) P_{z}\right)}{\sqrt{2 \pi} C_{33} C_{44} u_{1}\left(u_{1}^{2}-u_{2}^{2}\right)}, \\
C_{3}, C_{4}= & \frac{-4\left(\left(\frac{1}{4} C_{33}\left(k \pm u_{2}\right)^{2}-\frac{1}{2} C_{33} k\left(k \pm u_{2}\right)-\alpha^{2} C_{44}\right) P_{x}-i \alpha\left(\frac{1}{2}\left(C_{13}+C_{44}\right)\left(k \pm u_{2}\right)-k C_{44}\right) P_{z}\right)}{\sqrt{2 \pi} C_{33} C_{44} u_{2}\left(u_{1}^{2}-u_{2}^{2}\right)}, \\
D_{1}, D_{2}= & \frac{-4\left(-i \alpha\left(\frac{1}{2}\left(C_{13}+C_{44}\right)\left(k \pm u_{1}\right)-k C_{13}\right) P_{x}+\left(\frac{1}{4} C_{44}\left(k \pm u_{1}\right)^{2}-\frac{1}{2} C_{44} k\left(k \pm u_{1}\right)-\alpha^{2} C_{11}\right) P_{z}\right)}{\sqrt{2 \pi} C_{33} C_{44} u_{1}\left(u_{1}^{2}-u_{2}^{2}\right)}, \\
D_{3}, D_{4}= & \frac{-4\left(-i \alpha\left(\frac{1}{2}\left(C_{13}+C_{44}\right)\left(k \pm u_{2}\right)-k C_{13}\right) P_{x}+\left(\frac{1}{4} C_{44}\left(k \pm u_{2}\right)^{2}-\frac{1}{2} C_{44} k\left(k \pm u_{2}\right)-\alpha^{2} C_{11}\right) P_{z}\right)}{\sqrt{2 \pi} C_{33} C_{44} u_{2}\left(u_{1}^{2}-u_{2}^{2}\right)} .
\end{aligned}
$$

The general solutions are obtained as sums of homogeneous and particular solutions:

- For $z \geq 0$ :

$$
\begin{aligned}
& \bar{u}_{x}(G)=\left(A_{1}+C_{1}\right) e^{\left(k+u_{1}\right) z / 2}+\left(A_{2}+C_{2}\right) e^{\left(k-u_{1}\right) z / 2}+\left(A_{3}+C_{3}\right) e^{\left(k+u_{2}\right) z / 2}+\left(A_{4}+C_{4}\right) e^{\left(k-u_{2}\right) z / 2}, \\
& \bar{u}_{z}(G)=\left(S_{1} A_{1}+D_{1}\right) e^{\left(k+u_{1}\right) z / 2}+\left(S_{2} A_{2}+D_{2}\right) e^{\left(k-u_{1}\right) z / 2}+\left(S_{3} A_{3}+D_{3}\right) e^{\left(k+u_{2}\right) z / 2}+\left(S_{4} A_{4}+D_{4}\right) e^{\left(k-u_{2}\right) z / 2} .
\end{aligned}
$$


- For $z<0$ :

$$
\begin{aligned}
& \bar{u}_{x}(G)=A_{1} e^{\left(k+u_{1}\right) z / 2}+A_{2} e^{\left(k-u_{1}\right) z / 2}+A_{3} e^{\left(k+u_{2}\right) z / 2}+A_{4} e^{\left(k-u_{2}\right) z / 2}, \\
& \bar{u}_{z}(G)=S_{1} A_{1} e^{\left(k+u_{1}\right) z / 2}+S_{2} A_{2} e^{\left(k-u_{1}\right) z / 2}+S_{3} A_{3} e^{\left(k+u_{2}\right) z / 2}+S_{4} A_{4} e^{\left(k-u_{2}\right) z / 2} .
\end{aligned}
$$

In these four equations, we assume $k+u_{1}$ and $k+u_{2}$ are positive and $k-u_{1}$ and $k-u_{2}$ are negative, so we can utilize the two infinite boundary conditions $(z \rightarrow \infty$ and $-\infty)$ of displacements to solve for the undetermined constants $A_{i}$. The final expressions for the general solutions $\bar{u}_{x}(\alpha, z)$ and $\bar{u}_{z}(\alpha, z)$ in the Fourier domain are as follows:

- For $z \geq 0$ :

$$
\begin{aligned}
\bar{u}_{x}(\alpha, z) & =\frac{4}{\sqrt{2 \pi} C_{33} C_{44}\left(u_{1}^{2}-u_{2}^{2}\right)} \\
\times & \left(\left(\left(\frac{1}{4} C_{33}\left(k-u_{1}\right)^{2}-\frac{1}{2} C_{33} k\left(k-u_{1}\right)-\alpha^{2} C_{44}\right) P_{x}-i \alpha\left(\frac{1}{2}\left(C_{13}+C_{44}\right)\left(k-u_{1}\right)-k C_{44}\right) P_{z}\right) \frac{e^{\left(k-u_{1}\right) z / 2}}{u_{1}}\right. \\
& \left.-\left(\left(\frac{1}{4} C_{33}\left(k-u_{2}\right)^{2}-\frac{1}{2} C_{33} k\left(k-u_{2}\right)-\alpha^{2} C_{44}\right) P_{x}-i \alpha\left(\frac{1}{2}\left(C_{13}+C_{44}\right)\left(k-u_{2}\right)-k C_{44}\right) P_{z}\right) \frac{e^{\left(k-u_{2}\right) z / 2}}{u_{2}}\right),
\end{aligned}
$$

$$
\begin{aligned}
\bar{u}_{z}(\alpha, z) & =\frac{4}{\sqrt{2 \pi} C_{33} C_{44}\left(u_{1}^{2}-u_{2}^{2}\right)} \\
\times & \left(\left(-i \alpha\left(\frac{1}{2}\left(C_{13}+C_{44}\right)\left(k-u_{1}\right)-k C_{13}\right) P_{x}+\left(\frac{1}{4} C_{44}\left(k-u_{1}\right)^{2}-\frac{1}{2} C_{44} k\left(k-u_{1}\right)-\alpha^{2} C_{11}\right) P_{z}\right) \frac{e^{\left(k-u_{1}\right) z / 2}}{u_{1}}\right. \\
& \left.-\left(-i \alpha\left(\frac{1}{2}\left(C_{13}+C_{44}\right)\left(k-u_{2}\right)-k C_{13}\right) P_{x}+\left(\frac{1}{4} C_{44}\left(k-u_{2}\right)^{2}-\frac{1}{2} C_{44} k\left(k-u_{2}\right)-\alpha^{2} C_{11}\right) P_{z}\right) \frac{e^{\left(k-u_{2}\right) z / 2}}{u_{2}}\right)
\end{aligned}
$$

- For $z<0$ :

$$
\begin{aligned}
\bar{u}_{x}(\alpha, z) & =\frac{4}{\sqrt{2 \pi} C_{33} C_{44}\left(u_{1}^{2}-u_{2}^{2}\right)} \\
\times & \left(\left(\left(\frac{1}{4} C_{33}\left(k+u_{1}\right)^{2}-\frac{1}{2} C_{33} k\left(k+u_{1}\right)-\alpha^{2} C_{44}\right) P_{x}-i \alpha\left(\frac{1}{2}\left(C_{13}+C_{44}\right)\left(k+u_{1}\right)-k C_{44}\right) P_{z}\right) \frac{e^{\left(k+u_{1}\right) z / 2}}{u_{1}}\right. \\
& \left.-\left(\left(\frac{1}{4} C_{33}\left(k+u_{2}\right)^{2}-\frac{1}{2} C_{33} k\left(k+u_{2}\right)-\alpha^{2} C_{44}\right) P_{x}-i \alpha\left(\frac{1}{2}\left(C_{13}+C_{44}\right)\left(k+u_{2}\right)-k C_{44}\right) P_{z}\right) \frac{e^{\left(k+u_{2}\right) z / 2}}{u_{2}}\right),
\end{aligned}
$$

$$
\begin{aligned}
\bar{u}_{z}(\alpha, z)=\frac{4}{\sqrt{2 \pi} C_{33} C_{44}\left(u_{1}^{2}-u_{2}^{2}\right)} & \left(\frac{2 i \alpha\left[C_{13}\left(k-u_{1}\right)-C_{44}\left(k+u_{1}\right)\right]}{4 \alpha^{2} C_{44}+C_{33}\left(k^{2}-u_{1}^{2}\right)}\left(-Q_{x 1} P_{x}+i \alpha Q_{z 1} P_{z}\right) \frac{e^{\left(k+u_{1}\right) z / 2}}{u_{1}}\right. \\
& \left.-\frac{2 i \alpha\left[C_{13}\left(k-u_{2}\right)-C_{44}\left(k+u_{2}\right)\right]}{4 \alpha^{2} C_{44}+C_{33}\left(k^{2}-u_{2}^{2}\right)}\left(-Q_{x 2} P_{x}+i \alpha Q_{z 2} P_{z}\right) \frac{e^{\left(k+u_{2}\right) z / 2}}{u_{2}}\right),
\end{aligned}
$$

where in the last expression we used the abbreviations $Q_{x i}=\left(\frac{1}{4} C_{33}\left(k+u_{i}\right)^{2}-\frac{1}{2} C_{33} k\left(k+u_{i}\right)-\alpha^{2} C_{44}\right)$ and $Q_{z i}=\left(\frac{1}{2}\left(C_{13}+C_{44}\right)\left(k+u_{i}\right)-k C_{44}\right)$, for $i=1,2$. 
The desired solutions for the displacements $u_{x}(x, z)$ and $u_{z}(x, z)$ in the inhomogeneous plane strain cross-anisotropic full space can be obtained by taking the inverse Fourier transforms with respect to $\alpha$ :

$$
\left[\begin{array}{l}
u_{x}(x, z) \\
u_{z}(x, z)
\end{array}\right]=\frac{1}{\sqrt{2 \pi}} \int_{-\infty}^{\infty}\left[\begin{array}{l}
\bar{u}_{x}(\alpha, z) \\
\bar{u}_{z}(\alpha, z)
\end{array}\right] e^{i \alpha x} d \alpha
$$

\section{Fundamental solutions of stresses in the Fourier domain}

The horizontal normal stress $\bar{\sigma}_{x x}(\alpha, z)$, the vertical normal stress $\bar{\sigma}_{z z}(\alpha, z)$, and the shear stress $\bar{\tau}_{x z}(\alpha, z)$ can be derived by combining (1) and (3) and performing the Fourier transforms; the result is

$$
\begin{gathered}
\bar{\sigma}_{x x}(\alpha, z)=-i \alpha C_{11} \bar{u}_{x}(\alpha, z)-C_{13} \frac{d \bar{u}_{z}(\alpha, z)}{d z}, \quad \bar{\sigma}_{z z}(\alpha, z)=-i \alpha C_{13} \bar{u}_{x}(\alpha, z)-C_{33} \frac{d \bar{u}_{z}(\alpha, z)}{d z}, \\
\bar{\tau}_{x z}(\alpha, z)=-C_{44}\left(\frac{d \bar{u}_{x}(\alpha, z)}{d z}+i \alpha \bar{u}_{z}(\alpha, z)\right) .
\end{gathered}
$$

Thus, for example, the stresses for $z \geq 0$ are

$$
\begin{gathered}
\bar{\sigma}_{x x}(\alpha, z)=\frac{e^{\left(k-u_{1}-u_{2}\right) z / 2}}{2 \sqrt{2 \pi} C_{33} C_{44} u_{1} u_{2}\left(u_{1}^{2}-u_{2}^{2}\right)} \\
\times\left(2 i \alpha \left(-u_{1} e^{u_{1} z / 2}\left\{C_{13} C_{44}\left(k-u_{2}\right)^{2}-C_{13}^{2}\left(k^{2}-u_{2}^{2}\right)+C_{11}\left[4 \alpha^{2} C_{44}+C_{33}\left(k^{2}-u_{2}^{2}\right)\right]\right\}\right.\right. \\
\left.\quad+u_{2} e^{u_{2} z / 2}\left\{C_{13} C_{44}\left(k-u_{1}\right)^{2}-C_{13}^{2}\left(k^{2}-u_{1}^{2}\right)+C_{11}\left[4 \alpha^{2} C_{44}+C_{33}\left(k^{2}-u_{1}^{2}\right)\right]\right\}\right) P_{x} \\
+\left(-u_{1}\left(k+u_{2}\right) C_{44} e^{u_{1} z / 2}\left[4 \alpha^{2} C_{11}+C_{13}\left(k-u_{2}\right)^{2}\right]\right. \\
\left.\left.+u_{2}\left(k+u_{1}\right) C_{44} e^{u_{2} z / 2}\left[4 \alpha^{2} C_{11}+C_{13}\left(k-u_{1}\right)^{2}\right]\right) P_{z}\right),
\end{gathered}
$$

$$
\begin{gathered}
\bar{\sigma}_{z z}(\alpha, z)=\frac{e^{\left(k-u_{1}-u_{2}\right) z / 2}}{2 \sqrt{2 \pi} C_{33} C_{44} u_{1} u_{2}\left(u_{1}^{2}-u_{2}^{2}\right)} \times\left(\begin{array}{c}
2 i \alpha\left(-u_{1} C_{44} e^{u_{1} z / 2}\left[4 \alpha^{2} C_{13}+C_{33}\left(k-u_{2}\right)^{2}\right]\right. \\
\left.+u_{2} C_{44} e^{u_{2} z / 2}\left[4 \alpha^{2} C_{13}+C_{33}\left(k-u_{1}\right)^{2}\right]\right) P_{x}
\end{array}\right. \\
+\left(-u_{1} e^{u_{1} z / 2}\left\{-4 \alpha^{2} C_{13}^{2}\left(k-u_{2}\right)+4 \alpha^{2} C_{13} C_{44}\left(k+u_{2}\right)+C_{33}\left(k-u_{2}\right)\left[4 \alpha^{2} C_{11}+C_{44}\left(k^{2}-u_{2}^{2}\right)\right]\right\}\right. \\
\left.\left.+u_{2} e^{u_{2} z / 2}\left\{-4 \alpha^{2} C_{13}^{2}\left(k-u_{1}\right)+4 \alpha^{2} C_{13} C_{44}\left(k+u_{1}\right)+C_{33}\left(k-u_{1}\right)\left[4 \alpha^{2} C_{11}+C_{44}\left(k^{2}-u_{1}^{2}\right)\right]\right\}\right) P_{z}\right),
\end{gathered}
$$

$$
\begin{aligned}
& \bar{\tau}_{x z}(\alpha, z)=\frac{e^{\left(k-u_{1}-u_{2}\right) z / 2}}{2 \sqrt{2 \pi} C_{33} u_{1} u_{2}\left(u_{1}^{2}-u_{2}^{2}\right)} \times\left(\left(-u_{1}\left(k+u_{2}\right) e^{u_{1} z / 2}\left[4 \alpha^{2} C_{13}+C_{33}\left(k-u_{2}\right)^{2}\right]\right.\right. \\
& \left.+u_{2}\left(k+u_{1}\right) e^{u_{2} z / 2}\left[4 \alpha^{2} C_{13}+C_{33}\left(k-u_{1}\right)^{2}\right]\right) P_{x} \\
& +2 i \alpha\left(-u_{1} e^{u_{1} z / 2}\left[4 \alpha^{2} C_{11}+C_{13}\left(k-u_{2}\right)^{2}\right]\right. \\
& \left.\left.+u_{2} e^{u_{2} z / 2}\left[4 \alpha^{2} C_{11}+C_{13}\left(k-u_{1}\right)^{2}\right]\right) P_{z}\right) .
\end{aligned}
$$

Finally, the stresses $\sigma_{x x}(x, z), \sigma_{z z}(x, z)$, and $\tau_{x z}(x, z)$ in the physical domain are determined by inverse Fourier transformation:

$$
\left[\begin{array}{c}
\sigma_{x x}(x, z) \\
\sigma_{z z}(x, z) \\
\tau_{x z}(x, z)
\end{array}\right]=\frac{1}{\sqrt{2 \pi}} \int_{-\infty}^{\infty}\left[\begin{array}{c}
\bar{\sigma}_{x x}(\alpha, z) \\
\bar{\sigma}_{z z}(\alpha, z) \\
\bar{\tau}_{x z}(\alpha, z)
\end{array}\right] e^{i \alpha x} d \alpha
$$


However, from (9) and (13), the formulation of displacements $u_{x}(x, z)$ and $u_{z}(x, z)$ and stresses $\sigma_{x x}(x, z), \sigma_{z z}(x, z)$, and $\tau_{x z}(x, z)$ in a continuously inhomogeneous cross-anisotropic full space have very complicated integrals, which cannot be given in an exact closed form; hence, numerical inversion of the Fourier transforms is required. The detailed numerical integrations required to estimate the vertical normal stress are elucidated in the next section.

\section{An illustrative example}

From (10)-(13), the numerical inversion of Fourier transforms for stress fields can be performed by using the routines of QUADPACK. QUADPACK can be utilized for the numerical computation of definite one-dimensional integrals [Piessens et al. 1983; Barros and Mesquita 1999]. There are routines for adaptive and nonadaptive integration, finite, semiinfinite and fully infinite integration regions, integrands with singularities, and integrands with $\sin x$ or $\cos x$. Therefore, the values of (13) can be accurately calculated by employing the numerical integration subroutine QDAGI between the limits $-\infty$ and $\infty$. In this investigation, the displacements, (9), owing to horizontal/vertical line loadings in the full space are only meaningful if evaluated as the displacements of one point relative to another point, with both points located neither at the origin of loading nor at infinity [Poulos and Davis 1974]. Hence, in this section, a parametric study is conducted to clarify the effect of inhomogeneity, the type and degree of material anisotropy, and the loading types on the most interesting quantity in geotechnical engineering, namely, the vertical normal stress $\left(\sigma_{z z}\right)$.

Two illustrative examples, horizontal and vertical line loads acting in the inhomogeneous plane strain cross-anisotropic full space, are given to show the effect of the nondimensional inhomogeneity parameter $k z$; the degree of material anisotropy specified by the ratios $E / E^{\prime}, G^{\prime} / E^{\prime}$, and $\nu / v^{\prime}$; and the loading types $P_{x} / P_{z}$ (force/length) on $\sigma_{z z}$, respectively. In this investigation, the values of the nondimensional inhomogeneity parameter $k z$ ( $z$ is downward positive in this numerical analysis) are theoretically assumed to be $-0.3,-0.2,-0.1$, and 0 (homogeneous). As mentioned previously, the situation $k z<0$ $(-0.3,-0.2,-0.1)$ corresponds to soft surface, where $E, E^{\prime}$, and $G^{\prime}$ increase with the depth. This situation would be suitable for the earth materials, such as soils and rocks. Regarding the typical ranges of cross-anisotropic parameters, Gazetas [1982] summarized experimental data for deformational crossanisotropy of clays and sands. He concluded that the ratio $E / E^{\prime}$ for clays ranges from 0.6 to 4 , and was as low as 0.2 for sands. However, for the heavily overconsolidated London clay, the range of $E / E^{\prime}$ was 1.35-2.37, and that of the ratio $G^{\prime} / E^{\prime}$ was $0.23-0.44$ [Ward et al. 1965; Gibson 1974; Lee and Rowe 1989; Tarn and Lu 1991; Wang et al. 2008]. The ratio $v / v^{\prime}$ is hypothetically assumed to be within the range $0.75-1.5$ in this study. Therefore, the anisotropic ratios $\left(E / E^{\prime}, G^{\prime} / E^{\prime}\right.$, and $\left.v / \nu^{\prime}\right)$ of elastic constants for seven different soils are listed in Table 2.

The influence of inhomogeneity and of the degree and type of soil anisotropy on the vertical normal stress due to a horizontal line load acting in the cross-anisotropic material is explored first. Figure 2 plots the effect of the nondimensional inhomogeneity parameter $k z$ (equal to $-0.3,-0.2,-0.1$, or 0 ) on the nondimensional vertical normal stress $\left(z \sigma_{z z} / P_{x}\right)$ versus the nondimensional horizontal distance $(x / z)$, induced by a horizontal line load $\left(P_{x}\right)$ for isotropic soil 1 and for each of the cross-anisotropic soils 2-7. In order to examine the accuracy of the numerical integration by using QDAGI, comparisons are carried out with the extension of the homogeneous point load solutions of [Wang and Liao 1999] $(k z=0)$. That 


\begin{tabular}{|clll|cccl|}
\hline Soil type & $E / E^{\prime}$ & $G^{\prime} / E^{\prime}$ & $v / \nu^{\prime}$ & Soil type & $E / E^{\prime}$ & $G^{\prime} / E^{\prime}$ & $v / v^{\prime}$ \\
1 & 1 & 0.385 & 1 & & & & \\
2 & 1.35 & 0.385 & 1 & 5 & 1 & 0.44 & 1 \\
3 & 2.37 & 0.385 & 1 & 6 & 1 & 0.385 & 0.75 \\
4 & 1 & 0.23 & 1 & 7 & 1 & 0.385 & 1.5 \\
\hline
\end{tabular}

Table 2. Anisotropic ratios of elastic constants for different soil types. Soil 1 is isotropic, while the others exhibit cross-anisotropy. In each case, $E=50 \mathrm{MPa}$ and $v=0.3$.
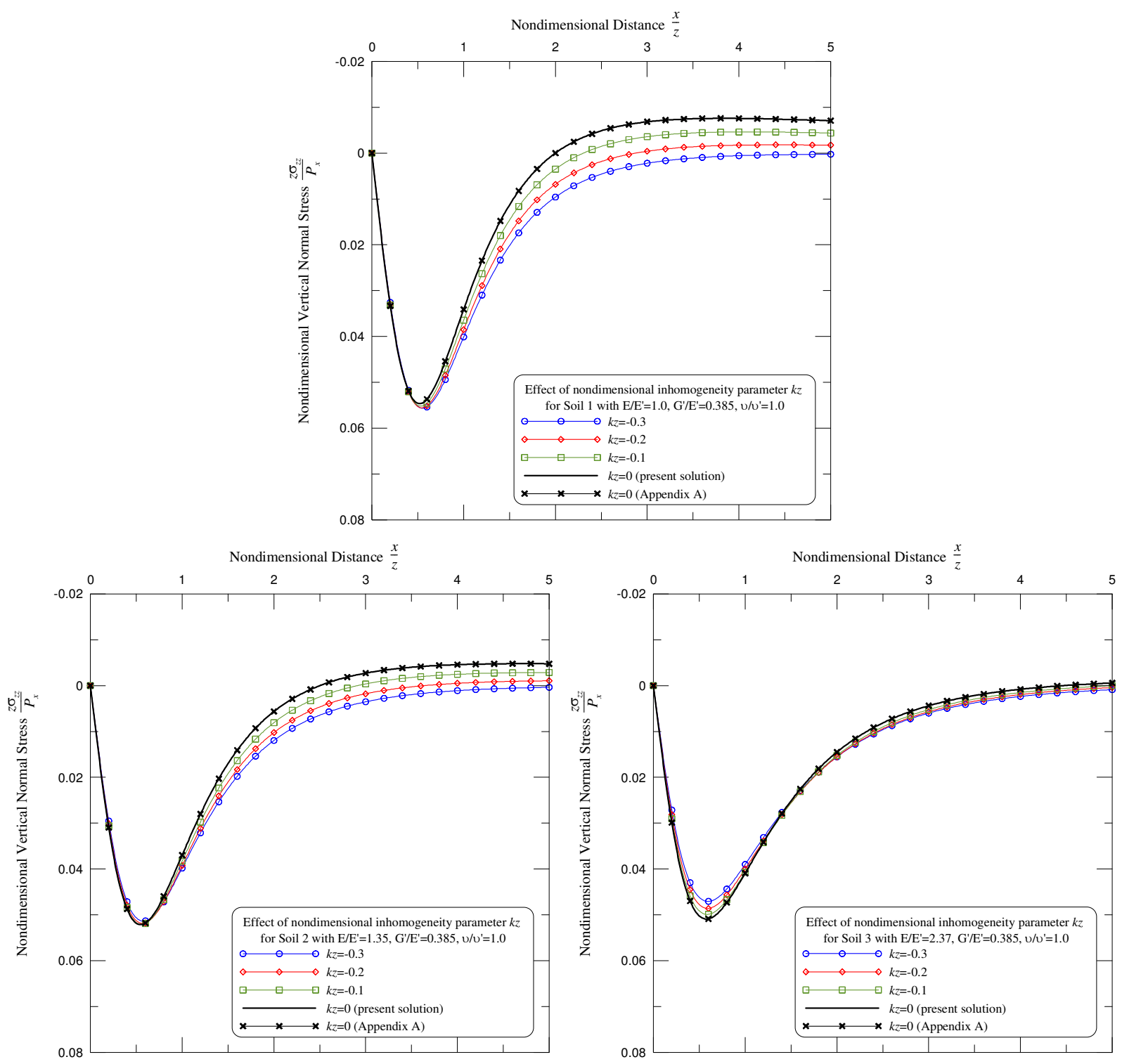

Figure 2. Effect of the inhomogeneity parameter $k z$ on the vertical normal stress $\left(\sigma_{z z}\right)$ induced by a horizontal line load $\left(P_{x}\right)$ for soil types $1-3$. (Continued on next page.) 
means the horizontal/vertical point load solutions for the stress components of [Wang and Liao 1999] in a cartesian coordinate system for a homogeneous cross-anisotropic full space should be extended to those of line load solutions by suitable integration. The new solutions are also expressed in the Appendix, and formulae (A.1)-(A.3) are the checking criteria for the present solutions, (10)-(13), in the case of the nondimensional inhomogeneity parameter $k z=0$. From Figure 2, the calculated results from the present solutions for soils 1-7 are in excellent agreement with those of in the Appendix. In addition, we see that when the nondimensional horizontal distance $x / z$ is less than 0.5 , the nondimensional vertical normal stresses $\left(z \sigma_{z z} / P_{x}\right)$ are almost the same irrespective of the variation of $k z$ for the isotropic medium
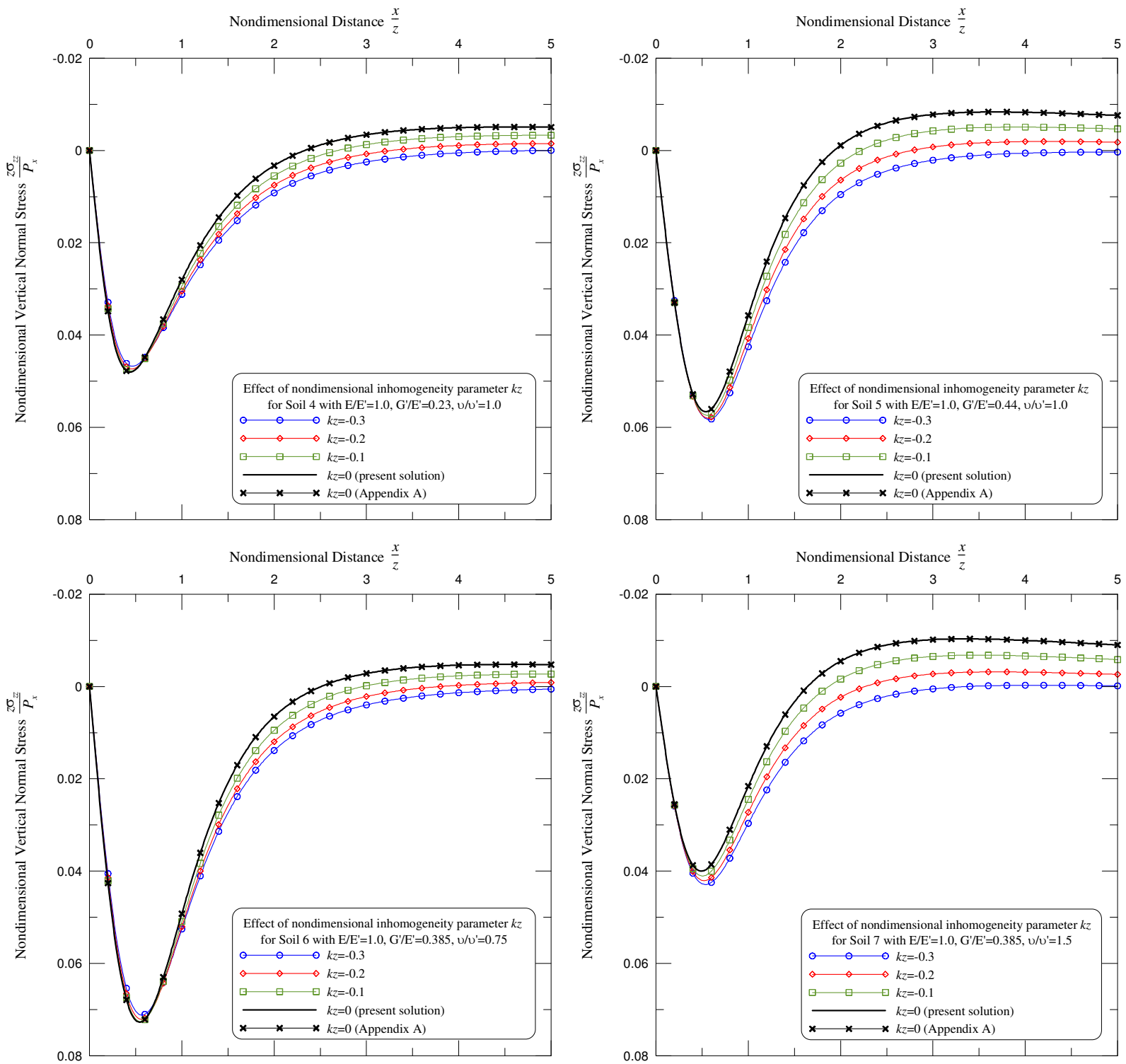

Figure 2 (continued). Effect of the inhomogeneity parameter $k z$ on the vertical normal stress $\left(\sigma_{z z}\right)$ induced by a horizontal line load $\left(P_{x}\right)$ for soil types 4-7. 
(soil 1). However, with increasing $x / z>1.5$, the vertical normal stress would be transferred by tension except for $k z=-0.3$, and the induced stress follows, decreasing steadily as $k z$ goes from -0.3 to 0 . It is apparent that the nondimensional inhomogeneity parameter $k z$ does have a great influence on $\sigma_{z z}$.

With an increase in $E / E^{\prime}$ from 1 for soil 1 to 1.35 for soil 2 and then to 2.37 for soil 3, the magnitude and region of the induced tensile stress is gradually reduced. In each figure, $z \sigma_{z z} / P_{x}$ converges with increasing $x / z$.

A similar trend, except for the magnitude, holds for the comparison of soils 1,4 and 5, with $G^{\prime} / E^{\prime}$ decreasing from 0.385 for soil 1 to 0.23 for soil 4 , and increasing to 0.44 for soil 5 .

However, $z \sigma_{z z} / P_{x}$ becomes larger within the very small loaded distance of $x / z(<1)$ with a decrease in $v / v^{\prime}$ from 1 for soil 1 , to 0.75 for soil 6 , and becomes smaller in the case of increasing $v / v^{\prime}$ (from 1 for soil 1 to 1.5 for soil 7). Thus, it is clear from Figure 2 that the induced vertical normal stress by a horizontal line load in a plane strain cross-anisotropic material strongly relies on the nondimensional inhomogeneity parameter $k z$ and the nondimensional horizontal distance $x / z$.

Figures 3-5 depict the effect of soil anisotropy $\left(E / E^{\prime}, G^{\prime} / E^{\prime}\right.$, and $\left.v / v^{\prime}\right)$ on the vertical normal stress $\left(\sigma_{z z}\right)$ induced by a horizontal line load $\left(P_{x}\right)$ for three groups of soils: 1,2 , and 3 ( $E / E^{\prime}$ varies, Figure 3$)$; soils 1,4 , and $5\left(G^{\prime} / E^{\prime}\right.$ varies, Figure 4$)$; and soils 1,6 , and 7 ( $v / v^{\prime}$ varies, Figure 5).

In each figure the cases $k z=-0.3\left(E, E^{\prime}\right.$, and $G^{\prime}$ increase with depth) and $k z=0$ (homogeneous) are shown, in the left and right panes respectively. From Figure 3, left, we see that for $x / z<1$, the order of the induced compression stress is soil $1>$ soil $2>$ soil 3 . However, with an increase of $x / z$, that order would be reversed.

In Figure 4, left, we see that the magnitudes of $z \sigma_{z z} / P_{x}$ obey the order soil $5>$ soil $1>$ soil 4 , while the corresponding order for Figure 5, left is soil $6>$ soil $1>$ soil 7, with a slight tensile stress observed in the case of soil 7 .
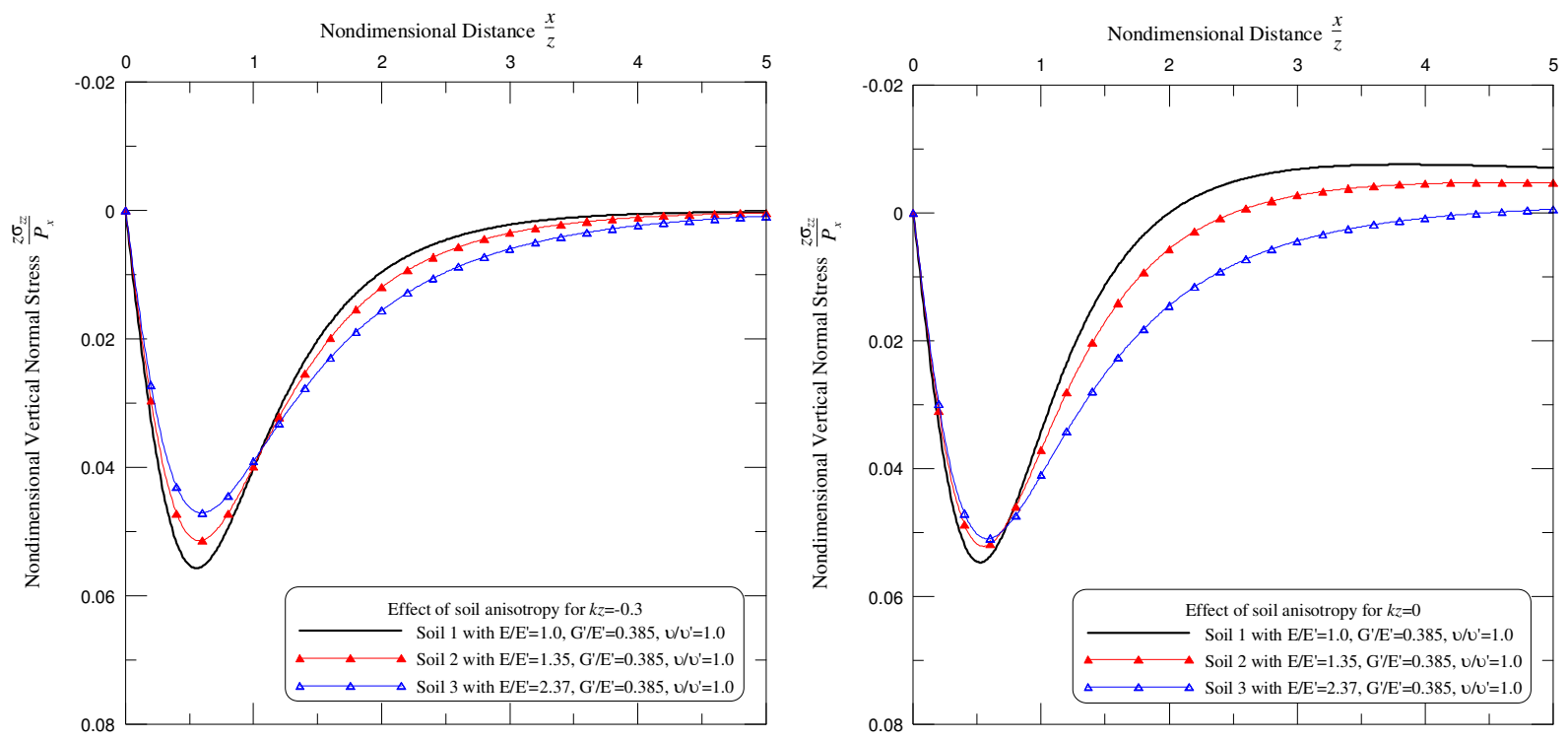

Figure 3. Effect of ratio $E / E^{\prime}$ on the vertical normal stress $\left(\sigma_{z z}\right)$ induced by a horizontal line load $\left(P_{x}\right)$ when $k z=-0.3$ (left) and $k z=0$ (right). Comparison of soils 1,2 , and 3 . 

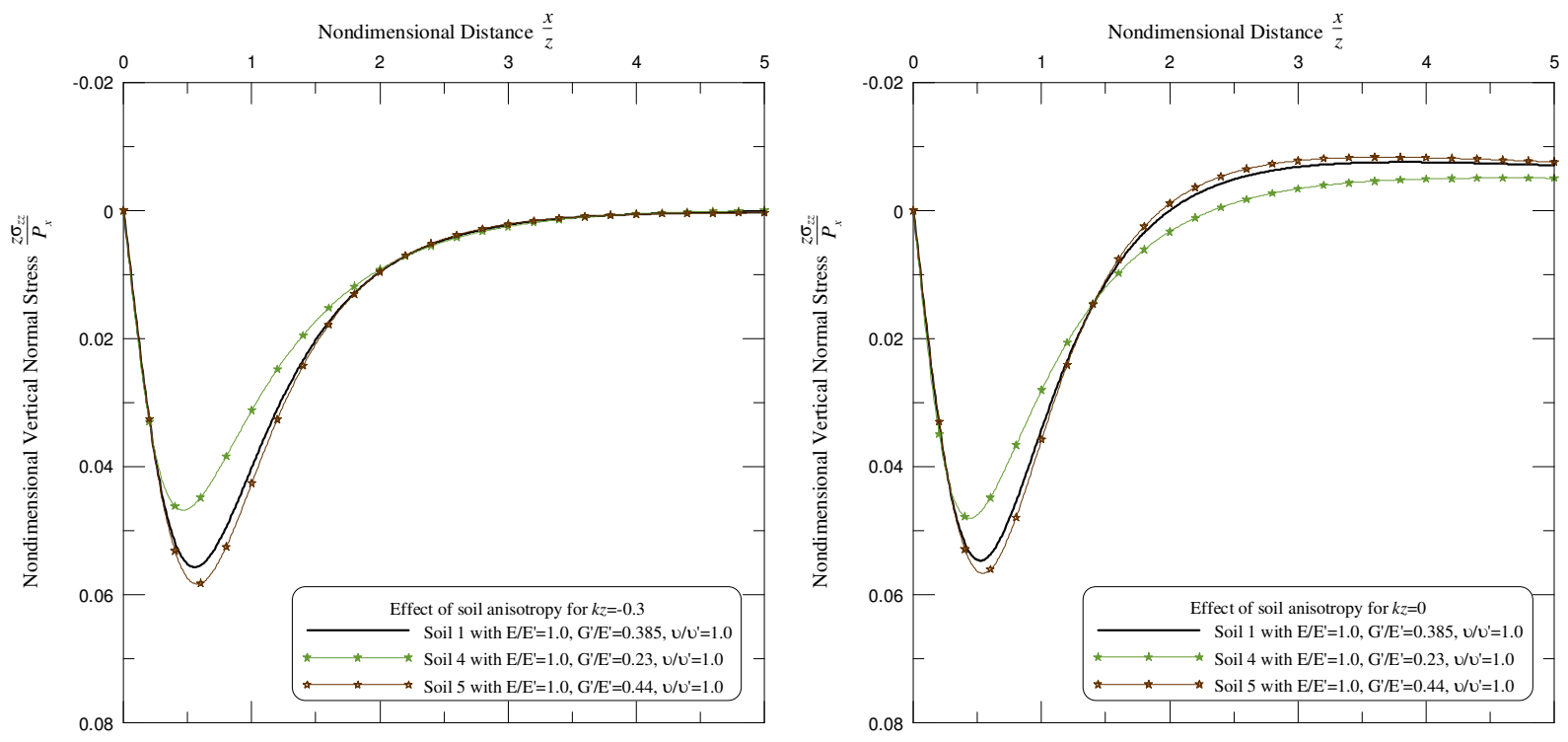

Figure 4. Effect of ratio $G^{\prime} / E^{\prime}$ on the vertical normal stress $\left(\sigma_{z z}\right)$ induced by a horizontal line load $\left(P_{x}\right)$ when $k z=-0.3$ (left) and $k z=0$ (right). Comparison of soils 1,4 , and 5 .
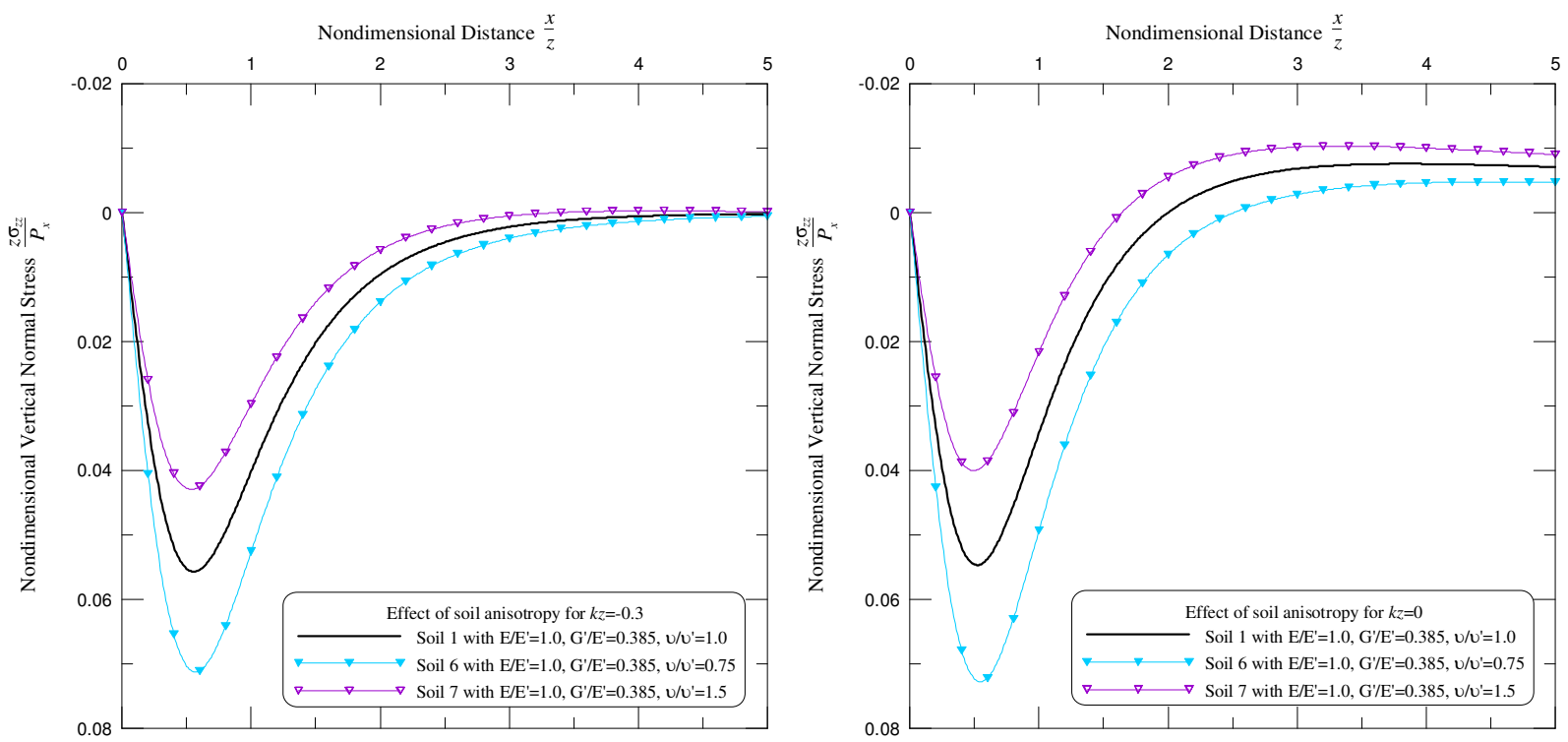

Figure 5. Effect of ratio $v / v^{\prime}$ on the vertical normal stress $\left(\sigma_{z z}\right)$ induced by a horizontal line load $\left(P_{x}\right)$ when $k z=-0.3$ (left) and $k z=0$ (right). Comparison of soils 1, 6, and 7 .

The right halves of Figures 3-5 represent the homogeneous case, $k z=0$. The trends here are quite different from those in the case $k z=-0.3$, especially in that a great deal of tension stress is produced in the homogeneous isotropic/cross-anisotropic media. 
From Figures 3-5 it is clear that the type and degree of soil anisotropy $\left(E / E^{\prime}, G^{\prime} / E^{\prime}\right.$, and $\left.v / v^{\prime}\right)$ affects profoundly the vertical normal stress due to a horizontal line load in an inhomogeneous isotropic/crossanisotropic material.

Figure 6 shows the effect of the nondimensional inhomogeneity parameter $k z$ (equal to $-0.3,-0.2$, -0.1 , or 0$)$ on the nondimensional vertical normal stress $\left(z \sigma_{z z} / P_{z}\right)$ versus the nondimensional horizontal distance $(x / z)$, subjected to a vertical line load $\left(P_{z}\right)$ for isotropic soil 1 and cross-anisotropic soils $2-7$. The figure suggests that the results of the present solutions when $k z=0$ for soils 1-7 agree very well with those in the Appendix. Additionally, we see that the vertical normal stresses due to a vertical line
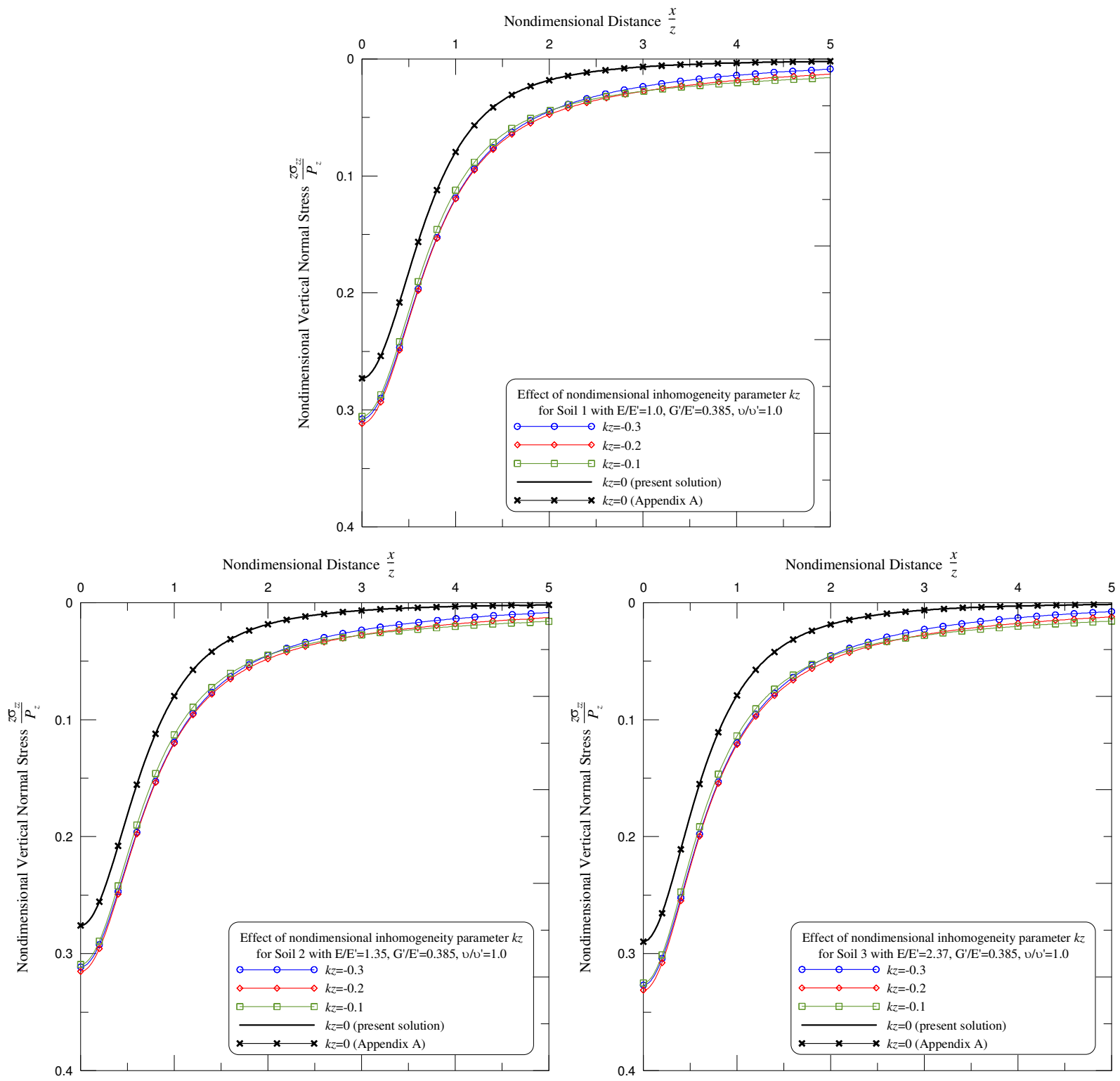

Figure 6. Effect of the inhomogeneity parameter $k z$ on the vertical normal stress $\left(\sigma_{z z}\right)$ induced by a vertical line load $\left(P_{z}\right)$ for soil types $1-3$. (Continued on next page.) 
load are all compressive, and these phenomena are different from those in Figure 2 for the stresses owing to a horizontal line load.

It can be seen that the magnitudes of $\sigma_{z z}$ induced by vertical line loading (Figure 6) are greater than those induced by horizontal line loading (Figure 2). Thus the type of loading $\left(P_{x}\right.$ or $\left.P_{z}\right)$ has a strong influence on $\sigma_{z z}$.

The plots for soils 1, 2 and 3 in Figure 6, with $E / E^{\prime}$ increasing from 1 to 1.35 and 2.37, all show the same trend, indicating that increases in $E / E^{\prime}$ (in this range) have only a minor effect on $\sigma_{z z}$.
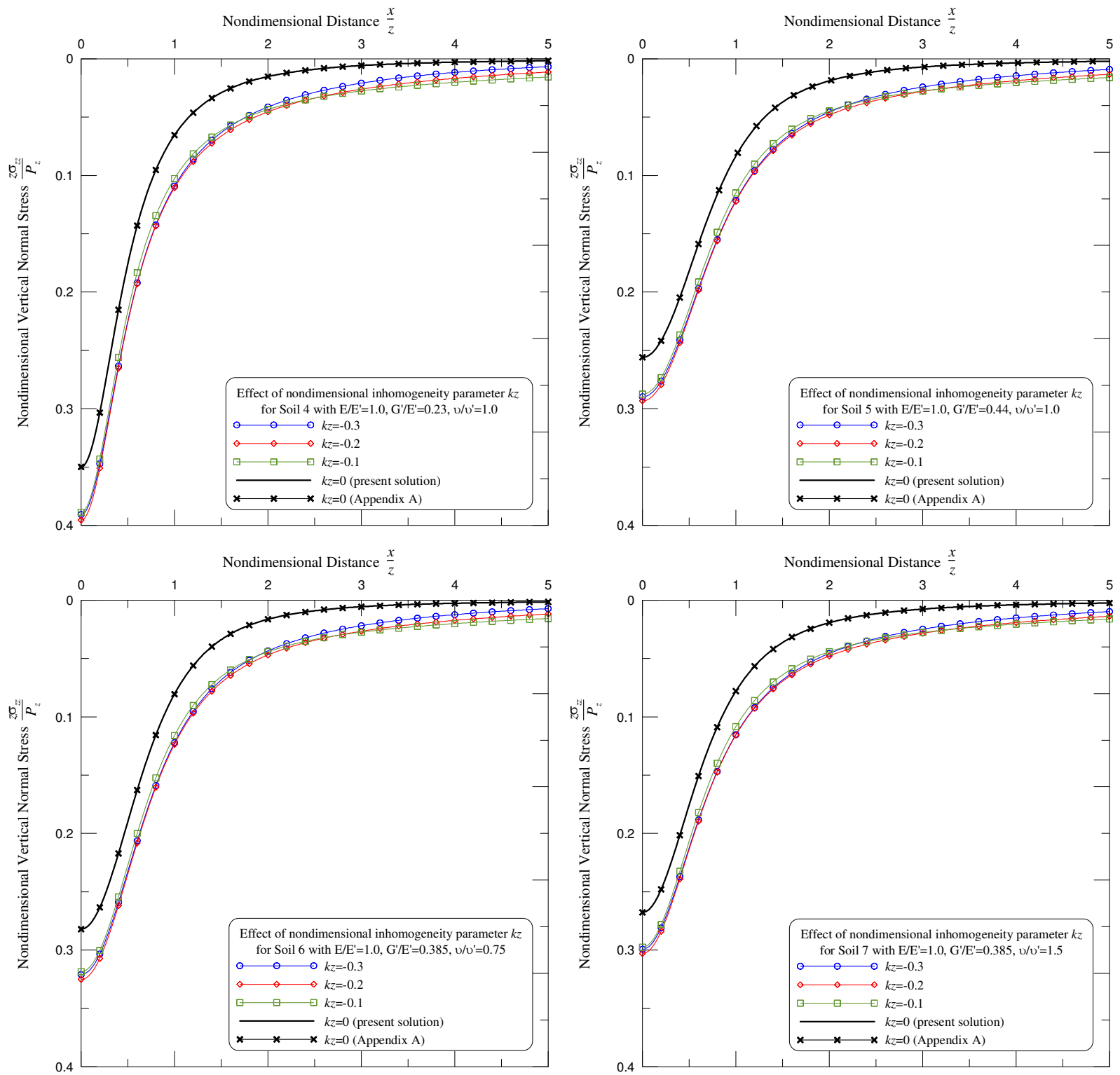

Figure 6 (continued). Effect of the inhomogeneity parameter $k z$ on the vertical normal stress $\left(\sigma_{z z}\right)$ induced by a vertical line load $\left(P_{z}\right)$ for soil types $4-7$. 
A comparison of the plots as $G^{\prime} / E^{\prime}$ decreases from 0.385 for soil 1 to 0.23 for soil 4 , and again as it increases to 0.44 for soil 5 , indicates that the ordering (relative to $k z$ ) of the graphs of $z \sigma_{z z} / P_{z}$ remains the same, but the magnitude changes significantly. The same is true for the decrease in $v / v^{\prime}$ from 1 for soil 1 to 0.75 for soil 6 , and its increase to 1.5 for soil 7 .

Figures 7-9 depict the effect of soil anisotropy $\left(E / E^{\prime}, G^{\prime} / E^{\prime}\right.$, and $\left.v / v^{\prime}\right)$ on the vertical normal stress $\left(\sigma_{z z}\right)$ induced by a vertical line load $\left(P_{z}\right)$ for three groups of soils: 1,2 , and $3\left(E / E^{\prime}\right.$ varies, Figure 7$)$; soils 1,4 , and $5\left(G^{\prime} / E^{\prime}\right.$ varies, Figure 8$)$; and soils 1,6 , and $7\left(v / v^{\prime}\right.$ varies, Figure 9$)$.

In each figure the cases $k z=-0.3\left(E, E^{\prime}\right.$, and $G^{\prime}$ increase with depth) and $k z=0$ (homogeneous) are shown, in the left and right panes respectively. It is seen that the induced vertical normal stresses are compressive for $k z=-0.3$ in all types of soil considered.

From Figure 7, left, we see that for $x / z<0.5$, the value of $z \sigma_{z z} / P_{z}$ increases as $E / E^{\prime}$ grows from 1 to 1.35 to 2.37; however, with higher $x / z$ from 0.5 to $2, z \sigma_{z z} / P_{z}$ in soils 1,2 , and 3 moves toward the same values. In Figure 8, left, $z \sigma_{z z} / P_{z}$ increases as $G^{\prime} / E^{\prime}$ drops from 0.385 to 0.23 , and decreases as $G^{\prime} / E^{\prime}$ grows from 0.385 to 0.44 , within the range $x / z<0.5$. It also can be found in Figure 9 , left, that $z \sigma_{z z} / P_{z}$ increases with a drop in $v / v^{\prime}$ from 1 to 0.75 , and decreases with an increase in $v / v^{\prime}$ from 1 to 1.5 , at about $x / z<1.2$.

The homogeneous case $(k z=0)$ is shown in the right-hand halves of Figures 7-9. When comparing this with the case $k z=-0.3$, we see that except for the magnitudes of $z \sigma_{z z} / P_{z}$ being diverse, the trends are similar. Overall, Figures 7-9 suggest that the magnitudes of vertical normal stresses are decisively influenced by soil anisotropy owing to a vertical line load in the isotropic/cross-anisotropic materials.

Moreover, it is evident from Figures 2-9 that the isotropic/cross-anisotropic materials resulting from different loading types $\left(P_{x}\right.$ and $\left.P_{z}\right)$ would affect the vertical normal stress. Therefore, we choose crossanisotropic soil $6\left(v / v^{\prime}=0.75\right)$ to interpret the effects of both loading types on $\sigma_{z z}$. Figure 10 shows
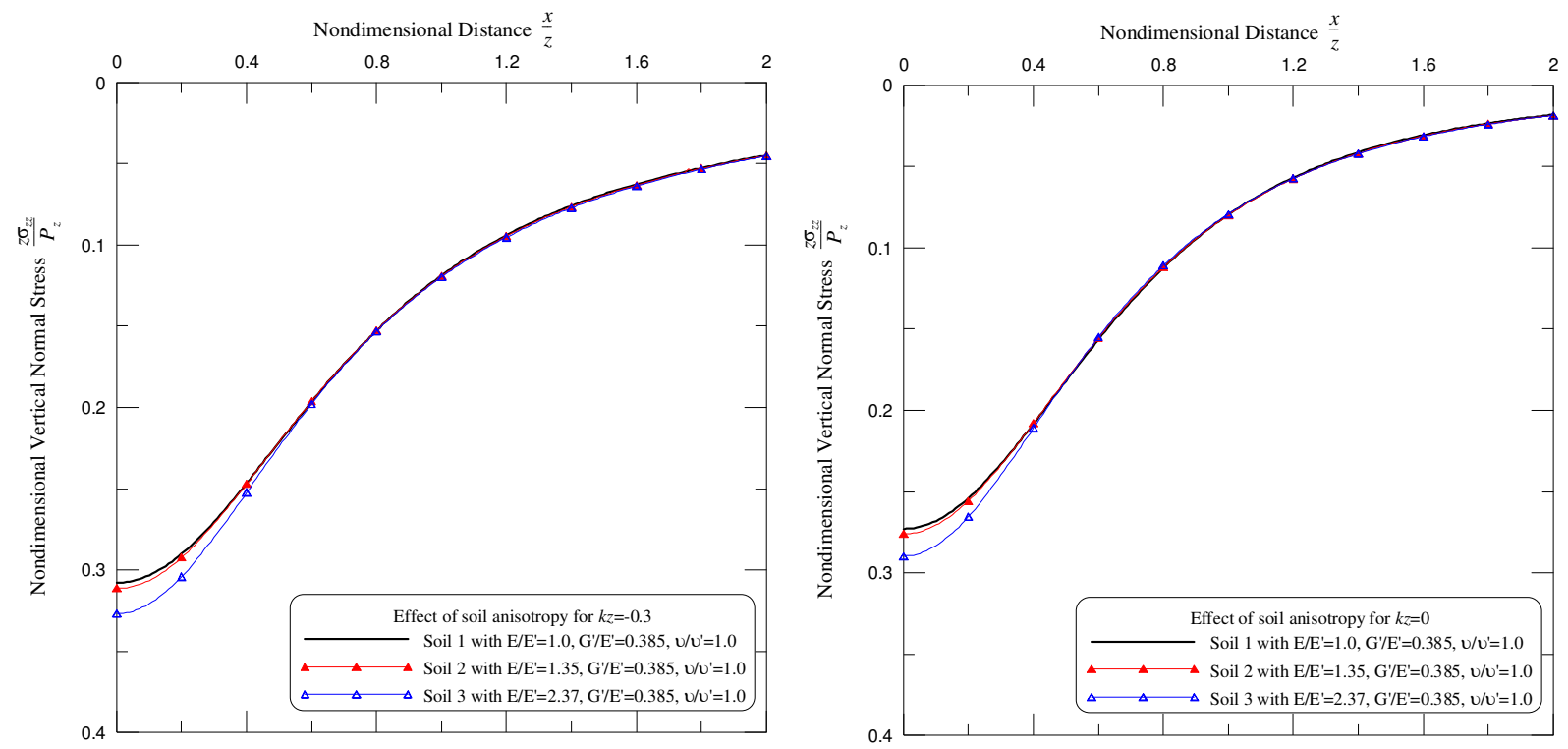

Figure 7. Effect of ratio $E / E^{\prime}$ on the vertical normal stress $\left(\sigma_{z z}\right)$ induced by a vertical line load $\left(P_{z}\right)$ when $k z=-0.3$ (left) and $k z=0$ (right). Comparison of soils 1, 2, and 3. 

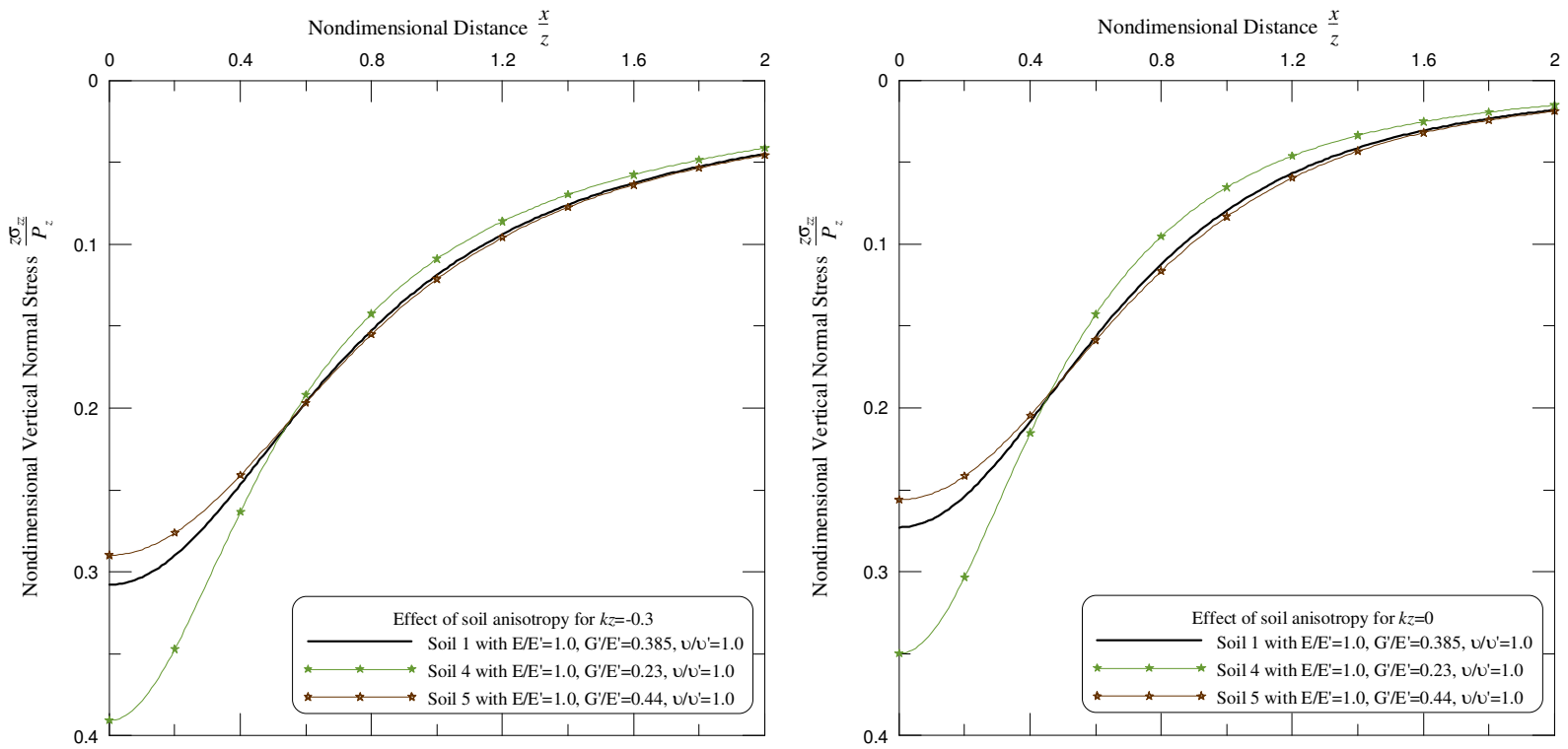

Figure 8. Effect of ratio $G^{\prime} / E^{\prime}$ on the vertical normal stress $\left(\sigma_{z z}\right)$ induced by a vertical line load $\left(P_{z}\right)$ when $k z=-0.3$ (left) and $k z=0$ (right). Comparison of soils 1,4 , and 5 .
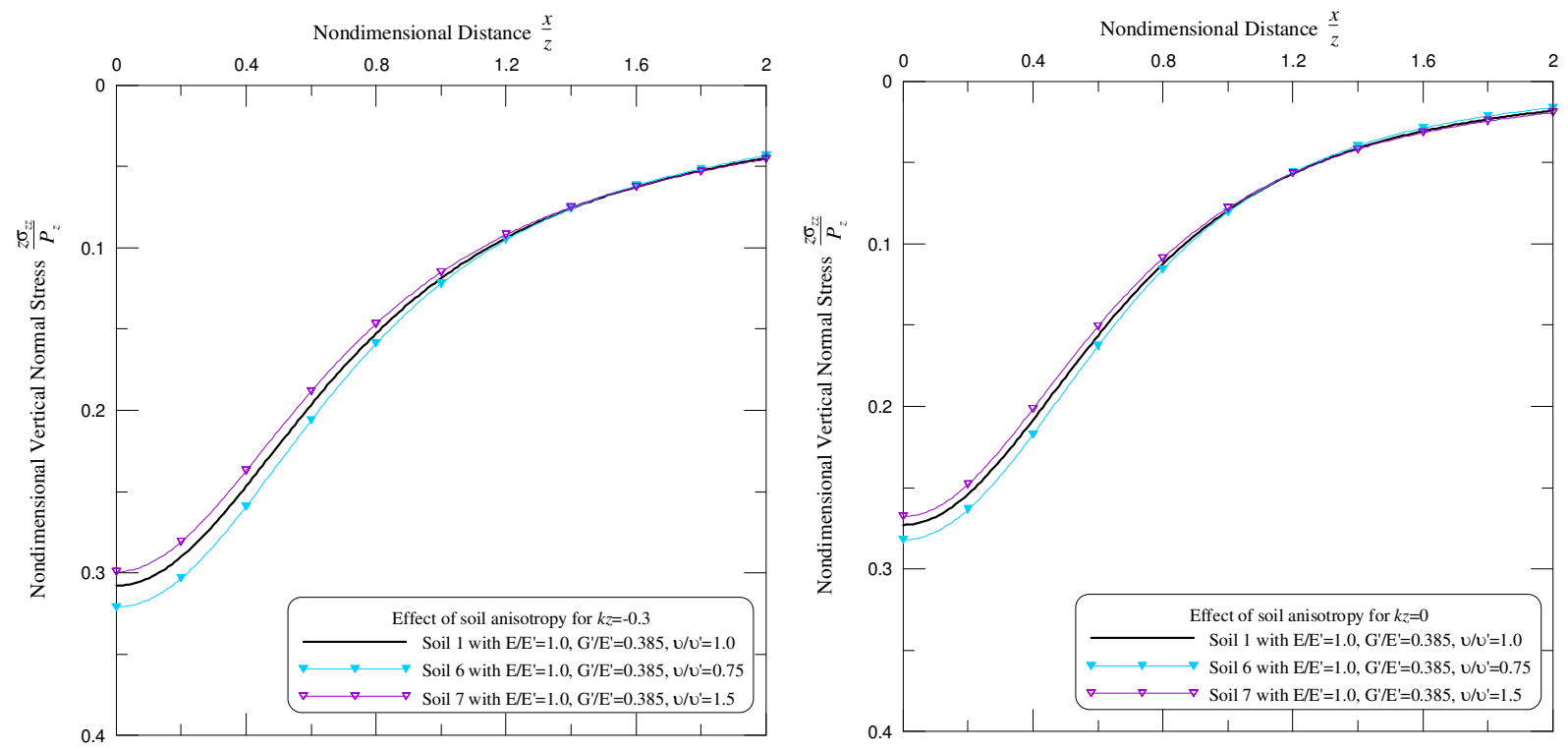

Figure 9. Effect of ratio $v / v^{\prime}$ on the vertical normal stress $\left(\sigma_{z z}\right)$ induced by a vertical line load $\left(P_{z}\right)$ when $k z=-0.3$ (left) and $k z=0$ (right). Comparison of soils 1,6 , and 7 .

the results for $k z=-0.3,-0.2,-0.1$, and 0 . We see that the induced vertical normal stresses are larger from a vertical line load $\left(P_{z}\right)$ than from a horizontal one $\left(P_{x}\right)$ in the case of $k z \leq 0$. Thus the type of loading deeply affect the vertical normal stress in inhomogeneous cross-anisotropic soil 6.

The examples above confirm the present solutions and clarify how the nondimensional inhomogeneity parameter $k z$, the type and degree of soil anisotropy, and the types of loading would influence the vertical 

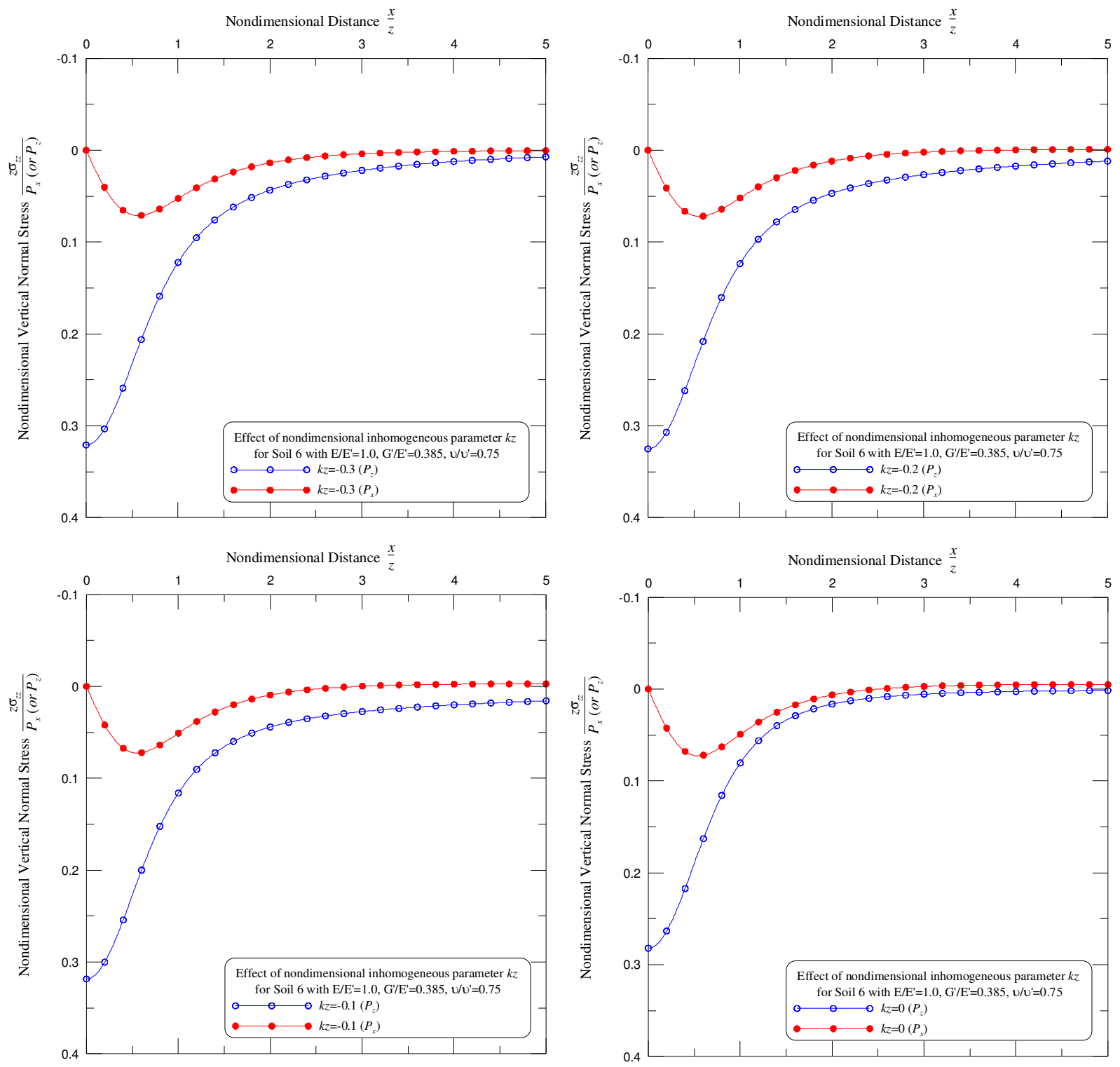

Figure 10. Effect of loading types $\left(P_{x}\right.$ and $\left.P_{z}\right)$ on the vertical normal stress $\left(\sigma_{z z}\right)$ for cross-anisotropic soil 6 for $k z=-0.3$ (top left), $-0.2,-0.1$, and 0 (bottom right).

normal stress, $\sigma_{z z}$, in the inhomogeneous plane strain isotropic/cross-anisotropic material. Numerical results also show that the stress in the continuously inhomogeneous plane strain cross-anisotropic full space resulting from horizontal and vertical line loads can be easily calculated by the proposed solutions. The magnitudes and distributions of vertical normal stress are simply sensitive to the nondimensional inhomogeneity parameter $k z$ (Figures 2 and 6), the anisotropic ratios specified by $E / E^{\prime}, G^{\prime} / E^{\prime}, v / v^{\prime}$ (Figures 3-5 and 7-9), and the different loading types (Figure 10). Also, the adopted nondimensional horizontal distance $x / z$ is another factor that could affect the present stress. Hence, the aforementioned 
factors should be taken into account when estimating the displacements and stresses in an inhomogeneous plane strain cross-anisotropic medium subjected to applied line loads.

\section{Conclusions}

The fundamental solutions of displacements and stresses in a cartesian coordinate system for a continuously inhomogeneous plane strain cross-anisotropic full space with Young's and shear moduli varying exponentially with depth, due to horizontal and vertical line loads, are derived in this article. The planes of cross-anisotropy are assumed to be horizontal. Fourier transforms with respect to $x$ are employed to find the solutions. However, inverse Fourier transforms for displacements and stresses are associated with complicated polynomial and exponential functions which cannot be given in exact closed forms; hence, numerical integration is required. The resulting integrals can be performed by the subroutine QDAGI between the limits $-\infty$ and $\infty$. The present solutions, setting the nondimensional inhomogeneity parameter $k z=0$, are then compared with the extension of Wang and Liao's horizontal/vertical point load solutions to horizontal/vertical infinite line load solutions for a homogeneous cross-anisotropic full space, as shown in the Appendix. The numerical results agree very well with those obtained in the Appendix. Overall, the generated solutions indicate that the displacements and stresses are deeply affected by all the factors considered: the nondimensional inhomogeneity parameter $(k z)$, the type and degree of material anisotropy $\left(E / E^{\prime}, G^{\prime} / E^{\prime}\right.$, and $\left.v / v^{\prime}\right)$, the types of loading $\left(P_{x}\right.$ and $\left.P_{z}\right)$, and the nondimensional horizontal distance $(x / z)$.

A series of parametric studies is conducted to demonstrate the present solutions, and elucidate the influence of aforementioned factors on the vertical normal stress. The results reveal that the computations of stresses (or displacements) should consider inhomogeneous characteristics in a cross-anisotropic material induced by applied line loads. In addition, these solutions could realistically imitate the actual stratum of loading problems in practical engineering. Most importantly, the proposed solutions can be used to derive the solutions of displacements and stresses resulting from the subjected line loads in an inhomogeneous plane strain cross-anisotropic half space; in particular, the actions of horizontal forces have never been provided in the literature. These matters will be addressed in forthcoming papers.

\section{Appendix}

The point load solutions of stress components for a homogeneous cross-anisotropic full space in a cartesian coordinate system can be rewritten from the solutions of [Wang and Liao 1999] as

$$
\begin{array}{r}
\sigma_{x x}^{p}=\frac{P_{x}}{4 \pi}\left[\left(C_{11}-u_{1}^{\prime} m_{1} C_{13}\right) g \frac{p_{11}}{m_{1}}-\left(C_{11}-u_{2}^{\prime} m_{2} C_{13}\right) g \frac{p_{12}}{m_{2}}-2 C_{66} g\left(\frac{p_{71}}{m_{1}}-\frac{p_{72}}{m_{2}}\right)+2 u_{3}^{\prime} p_{73}\right] \\
+\frac{P_{z}}{4 \pi} g\left[\left(C_{11}-u_{1}^{\prime} m_{1} C_{13}-2 C_{66}\right) p_{31}-\left(C_{11}-u_{2}^{\prime} m_{2} C_{13}-2 C_{66}\right) p_{32}+2 C_{66}\left(p_{51}-p_{52}\right)\right] \\
\sigma_{y y}^{p}=\frac{P_{x}}{4 \pi}\left[\left(C_{11}-u_{1}^{\prime} m_{1} C_{13}-2 C_{66}\right) g \frac{p_{11}}{m_{1}}-\left(C_{11}-u_{2}^{\prime} m_{2} C_{13}-2 C_{66}\right) g \frac{p_{12}}{m_{2}}+2 C_{66} g\left(\frac{p_{71}}{m_{1}}-\frac{p_{72}}{m_{2}}\right)-2 u_{3}^{\prime} p_{73}\right] \\
+\frac{P_{z}}{4 \pi} g\left[\left(C_{11}-u_{1}^{\prime} m_{1} C_{13}-2 C_{66}\right) p_{31}-\left(C_{11}-u_{2}^{\prime} m_{2} C_{13}-2 C_{66}\right) p_{32}+2 C_{66}\left(p_{61}-p_{62}\right)\right],
\end{array}
$$


$\sigma_{z z}^{p}=\frac{P_{x}}{4 \pi} g\left[\left(C_{13}-u_{1}^{\prime} m_{1} C_{33}\right) \frac{p_{11}}{m_{1}}-\left(C_{13}-u_{2}^{\prime} m_{2} C_{33}\right) \frac{p_{12}}{m_{2}}\right]$

$$
+\frac{P_{z}}{4 \pi} g\left[\left(C_{13}-u_{1}^{\prime} m_{1} C_{33}\right) p_{31}-\left(C_{13}-u_{2}^{\prime} m_{2} C_{33}\right) p_{32}\right],
$$

$\tau_{x y}^{p}=\frac{P_{x}}{4 \pi}\left[2 C_{66}\left(\frac{g}{m_{1}} p_{81}-\frac{g}{m_{2}} p_{82}\right)-u_{3}^{\prime}\left(2 p_{83}-p_{23}\right)\right]-\frac{P_{z}}{2 \pi} C_{66} g\left(p_{41}-p_{42}\right)$,

$\tau_{y z}^{p}=-\frac{P_{x}}{4 \pi}\left[\left(u_{1}^{\prime}+m_{1}\right) C_{44} \frac{g}{m_{1}} p_{41}-\left(u_{2}^{\prime}+m_{2}\right) C_{44} \frac{g}{m_{2}} p_{42}-p_{43}\right]-\frac{P_{z}}{4 \pi} g C_{44}\left[\left(u_{1}^{\prime}+m_{1}\right) p_{21}-\left(u_{2}^{\prime}+m_{2}\right) p_{22}\right]$,

$\tau_{x z}^{p}=\frac{P_{x}}{4 \pi}\left[\left(u_{1}^{\prime}+m_{1}\right) C_{44} \frac{g}{m_{1}} p_{51}-\left(u_{2}^{\prime}+m_{2}\right) C_{44} \frac{g}{m_{2}} p_{52}+p_{63}\right]-\frac{P_{z}}{4 \pi} g C_{44}\left[\left(u_{1}^{\prime}+m_{1}\right) p_{11}-\left(u_{2}^{\prime}+m_{2}\right) p_{12}\right]$,

where:

- $C_{11}, C_{13}, C_{33}$, and $C_{44}$ are the elastic moduli or elasticity constants of the cross-anisotropic material, as seen in (2); however, $C_{66}=E /(2(1+v))$.

- $u_{3}^{\prime}=\sqrt{C_{66} / C_{44}}, u_{1}^{\prime}$, and $u_{2}^{\prime}$ are the roots of the following characteristic equation: $u^{\prime 4}-s u^{\prime 2}+q=0$, where $s=\left(C_{11} C_{33}-C_{13}\left(C_{13}+2 C_{44}\right)\right) /\left(C_{33} C_{44}\right)$ and $q=C_{11} / C_{33}$.

Since the strain energy is assumed to be positive definite in the material, the values of the elastic constants are restricted. Hence, there are three categories of the characteristic roots, $u_{1}^{\prime}$ and $u_{2}^{\prime}$, as follows:

Case 1: $u_{1,2}^{\prime}= \pm \sqrt{\frac{1}{2}\left(s \pm \sqrt{s^{2}-4 q}\right)}$ are two real distinct roots when $s^{2}-4 q>0 ;$

Case 2: $u_{1,2}^{\prime}= \pm \sqrt{\frac{s}{2}}, \pm \sqrt{\frac{s}{2}}$ are double equal real roots when $s^{2}-4 q=0$;

Case 3: $u_{1}^{\prime}=\frac{1}{2} \sqrt{s+2 \sqrt{q}}-i \frac{1}{2} \sqrt{-s+2 \sqrt{q}}=\gamma-i \delta$ and $u_{2}^{\prime}=\gamma+i \delta$ are two complex conjugate roots (where $\gamma$ cannot be equal to zero) when $s^{2}-4 q<0$.

- $m_{j}=\frac{\left(C_{13}+C_{44}\right) u_{j}^{\prime}}{C_{33} u_{j}^{\prime 2}-C_{44}}=\frac{C_{11}-C_{44} u_{j}^{\prime 2}}{\left(C_{13}+C_{44}\right) u_{j}^{\prime}} \quad(j=1,2), \quad g=\frac{C_{13}+C_{44}}{C_{33} C_{44}\left(u_{1}^{\prime 2}-u_{2}^{\prime 2}\right)}$.

- Defining $p_{1 i}-p_{8 i}$ in (A.1)-(A.2) as the elementary functions, they can be represented as

$$
\begin{array}{rlrl}
p_{1 i} & =\frac{x}{R_{i}^{3}}, \quad p_{2 i}=\frac{y}{R_{i}^{3}}, & p_{3 i}=\frac{z_{i}}{R_{i}^{3}}, \quad p_{4 i}=\frac{x y\left(2 R_{i}+z_{i}\right)}{R_{i}^{3}\left(R_{i}+z_{i}\right)^{2}}, \\
p_{5 i}=\frac{1}{R_{i}\left(R_{i}+z_{i}\right)}-\frac{x^{2}\left(2 R_{i}+z_{i}\right)}{R_{i}^{3}\left(R_{i}+z_{i}\right)^{2}}, & p_{6 i}=\frac{1}{R_{i}\left(R_{i}+z_{i}\right)}-\frac{y^{2}\left(2 R_{i}+z_{i}\right)}{R_{i}^{3}\left(R_{i}+z_{i}\right)^{2}}, \\
p_{7 i}=\frac{x}{R_{i}^{3}}-\frac{3 x}{R_{i}\left(R_{i}+z_{i}\right)^{2}}+\frac{x^{3}\left(3 R_{i}+z_{i}\right)}{R_{i}^{3}\left(R_{i}+z_{i}\right)^{3}}, & p_{8 i}=\frac{y}{R_{i}^{3}}-\frac{3 y}{R_{i}\left(R_{i}+z_{i}\right)^{2}}+\frac{y^{3}\left(3 R_{i}+z_{i}\right)}{R_{i}^{3}\left(R_{i}+z_{i}\right)^{3}},
\end{array}
$$

and

$$
R_{i}=\sqrt{x^{2}+y^{2}+z_{i}^{2}}, \quad z_{i}=u_{i}^{\prime} z \quad(i=1,2,3) .
$$

The solutions for stresses in a homogeneous cross-anisotropic full space due to infinite horizontal and vertical line loads can be directly obtained by integrating the elementary functions of the point load solutions $\left(p_{1 i}-p_{8 i}\right)$. That is, the explicit solutions for stresses caused by horizontal and vertical infinite line loads in a full space can be regrouped in the form of (A.1)-(A.2). In other words, the exact 
solutions for the present case are the same as (A.1)-(A.2) except that the elementary functions, $p_{1 i}-p_{8 i}$ $(i=1,2,3)$, are replaced by the integral functions, $L_{1 i}-L_{8 i}(i=1,2,3)$, respectively. Therefore, only $L_{1 i}-L_{8 i}(i=1,2,3)$ are derived, and are given as

$$
\begin{aligned}
L_{1 i} & =\int_{-\infty}^{\infty} p_{1 i} d y=\frac{2 x}{x^{2}+z_{i}^{2}}, & L_{2 i} & =\int_{-\infty}^{\infty} p_{2 i} d y=0, \\
L_{3 i} & =\int_{-\infty}^{\infty} p_{3 i} d y=\frac{2 z_{i}}{x^{2}+z_{i}^{2}}, & L_{4 i} & =\int_{-\infty}^{\infty} p_{4 i} d y=0, \\
L_{5 i} & =\int_{-\infty}^{\infty} p_{5 i} d y=\frac{2 z_{i}}{x^{2}+z_{i}^{2}}, & L_{6 i} & =\int_{-\infty}^{\infty} p_{6 i} d y=0, \\
L_{7 i} & =\int_{-\infty}^{\infty} p_{7 i} d y=0, & L_{8 i} & =\int_{-\infty}^{\infty} p_{8 i} d y=0 .
\end{aligned}
$$

\section{References}

[Azis and Clements 2001] M. I. Azis and D. L. Clements, "A boundary element method for anisotropic inhomogeneous elasticity”, Int. J. Solids Struct. 38:32-33 (2001), 5747-5763.

[Barros and Mesquita 1999] P. L. A. Barros and E. de Mesquita Neto, "Elastodynamic Green's functions for orthotropic plane strain continua with inclined axes of symmetry”, Int. J. Solids Struct. 36:31-32 (1999), 4767-4788.

[Chen et al. 2001] L. Chen, A. J. Kassab, D. W. Nicholson, and M. B. Chopra, "Generalized boundary element method for solids exhibiting nonhomogeneities", Eng. Anal. Boundary Elem. 25:6 (2001), 407-422.

[Cihan 2003] O. A. Cihan, "Contact mechanics of a graded surface with elastic gradation in lateral direction", MS Thesis, The Middle East Technical University, Ankara, 2003.

[Davis and Rabinowitz 1984] P. J. Davis and P. Rabinowitz, Methods of numerical integration, 2nd ed., Academic Press, New York, 1984.

[Doherty and Deeks 2002] J. P. Doherty and A. J. Deeks, "Applications of the scaled boundary finite-element method to offshore geotechnical problems", pp. 706-711 in Twelfth International Offshore and Polar Engineering Conference, Kyushu, 2002.

[Doherty and Deeks 2003a] J. P. Doherty and A. J. Deeks, "Elastic response of circular footings embedded in a non-homogeneous half-space", Géotechnique 53:8 (2003), 703-714.

[Doherty and Deeks 2003b] J. P. Doherty and A. J. Deeks, "Scaled boundary finite-element analysis of a non-homogeneous axisymmetric domain subjected to general loading", Int. J. Numer. Anal. Meth. Geomech. 27:10 (2003), 813-835.

[Doherty and Deeks 2003c] J. P. Doherty and A. J. Deeks, "Scaled boundary finite-element analysis of a non-homogeneous elastic half-space", Int. J. Numer. Meth. Eng. 57:7 (2003), 955-973.

[Doherty and Deeks 2005] J. P. Doherty and A. J. Deeks, "Stiffness of flexible caisson foundations embedded in nonhomogeneous elastic soil", J. Geotech. Geoenvironmental Eng., ASCE 131:12 (2005), 1498-1508.

[Doherty and Deeks 2006] J. P. Doherty and A. J. Deeks, "Stiffness of a flexible circular footing embedded in an elastic half-space”, Int. J. Geomech., ASCE 6:1 (2006), 46-54.

[Gazetas 1982] G. Gazetas, "Stresses and displacements in cross-anisotropic soils", J. Geotech. Eng. Div., ASCE 108:4 (1982), $532-553$.

[Giannakopoulos and Suresh 1997] A. E. Giannakopoulos and S. Suresh, "Indentation of solids with gradients in elastic properties: Part I. Point force”, Int. J. Solids Struct. 34:19 (1997), 2357-2392.

[Gibson 1974] R. E. Gibson, "The analytical method in soil mechanics", Géotechnique 24:2 (1974), 115-140.

[Gray et al. 2001] L. J. Gray, J. D. Richardson, T. Kaplan, J. Berger, P. A. Martin, and G. H. Paulino, “Green's functions for exponentially graded elastic materials", pp. 327 in Sixth U.S. National Congress on Computational Mechanics, Dearborn, 2001. 
[Hu et al. 2007] T. B. Hu, C. D. Wang, and J. J. Liao, "Elastic solutions for a transversely isotropic full space with inclined planes of symmetry subjected to a point load", Int. J. Numer. Anal. Meth. Geomech. 31:12 (2007), 1401-1442.

[Lee and Rowe 1989] K. M. Lee and R. K. Rowe, "Deformation caused by surface loading and tunneling: the role of elastic anisotropy”, Géotechnique 39:1 (1989), 125-140.

[Longman 1956a] I. M. Longman, "Note on a method for computing infinite integrals of oscillatory functions", Proc. Cambridge Philos. Soc. 52 (1956), 764-768.

[Longman 1956b] I. M. Longman, "Tables for the rapid and accurate numerical evaluation of certain infinite integrals involving Bessel functions", Proc. Cambridge Philos. Soc. 52 (1956), 166-180.

[Martin 2004] P. A. Martin, "Fundamental solutions and functionally graded materials", in Seventh International Conference on Integral Methods in Science and Engineering (Saint-Étienne, 2002), edited by C. Constanda et al., Birkhäuser, Boston, 2004.

[Martin et al. 2002] P. A. Martin, J. D. Richardson, L. J. Gray, and J. R. Berger, “On Green's function for a three-dimensional exponentially graded elastic solid", Proc. Royal Soc. London A 458 (2002), 1931-1947.

[Piessens et al. 1983] R. Piessens, R. DeDoncker-Kapenga, C. W. Überhuber, and D. K. Kahaner, QUADPACK: a subroutine package for automatic integration, Springer, New York, 1983.

[Poulos and Davis 1974] G. H. Poulos and E. H. Davis, Elastic solutions for soil and rock mechanics, Wiley, New York, 1974.

[Tarn and Lu 1991] J. Q. Tarn and C. C. Lu, "Analysis of subsidence due to a point sink in an anisotropic porous elastic half space", Int. J. Numer. Anal. Meth. Geomech. 15:4 (1991), 573-592.

[Wang and Liao 1999] C. D. Wang and J. J. Liao, "Elastic solutions for a transversely isotropic half-space subjected to buried asymmetric-loads", Int. J. Numer. Anal. Meth. Geomech. 23:2 (1999), 115-139.

[Wang and Tzeng 2009] C. D. Wang and C. S. Tzeng, "Displacements and stresses induced by nonuniform circular loads in an inhomogeneous cross-anisotropic material", Mech. Res. Commun. 36 (2009), 921-932.

[Wang et al. 2003] C. D. Wang, C. S. Tzeng, E. Pan, and J. J. Liao, "Displacements and stresses due to a vertical point load in an inhomogeneous transversely isotropic half-space", Int. J. Rock Mech. Mining Sci. 40:5 (2003), 667-685.

[Wang et al. 2006] C. D. Wang, E. Pan, C. S. Tzeng, F. Han, and J. J. Liao, "Displacements and stresses due to a uniform vertical circular load in an inhomogeneous cross-anisotropic half-space", Int. J. Geomech., ASCE 6:1 (2006), 1-10.

[Wang et al. 2008] C. D. Wang, M. T. Chen, and T. C. Lee, "Surface displacements due to batter piles driven in cross-anisotropic media", Int. J. Numer. Anal. Meth. Geomech. 32:2 (2008), 121-141.

[Ward et al. 1965] W. H. Ward, A. Marsland, and S. G. Samuels, "Properties of the London clay at the ashford common shaft", Géotechnique 15:4 (1965), 321-344.

Received 13 Jan 2009. Revised 8 Aug 2009. Accepted 13 Aug 2009.

Cheng-Der WANG: cdwang@nuu.edu.tw

Department of Civil and Disaster Prevention Engineering, National United University, No. 1, Lien Da, Kung-Ching Li, Miao-Li, Taiwan 360, Taiwan

JiA-YAN HoU: ayanyan1982@yahoo.com.tw

Department of Civil and Disaster Prevention Engineering, National United University, No. 1, Lien Da, Kung-Ching Li,

Miao-Li, Taiwan 360, Taiwan

WeI-Jer WANG: wwj111@nuu.edu.tw

Department of Civil and Disaster Prevention Engineering, National United University, No. 1, Lien Da, Kung-Ching Li, Miao-Li, Taiwan 360, Taiwan 


\title{
JOURNAL OF MECHANICS OF MATERIALS AND STRUCTURES
}

\author{
http://www.jomms.org
}

\author{
Founded by Charles R. Steele and Marie-Louise Steele

\section{EDITORS} \\ Charles R. STEele \\ DAVIDE BIGONI \\ IWONA JASIUK \\ YASUHIDE SHINDO \\ Stanford University, U.S.A. \\ University of Trento, Italy \\ University of Illinois at Urbana-Champaign, U.S.A. \\ Tohoku University, Japan
}

\section{EDITORIAL BOARD}

H. D. BUI École Polytechnique, France

J. P. CARTER University of Sydney, Australia

R. M. Christensen Stanford University, U.S.A.

G. M. L. GLADWELL University of Waterloo, Canada

D. H. HodGES Georgia Institute of Technology, U.S.A.

J. HUTCHINSON Harvard University, U.S.A.

C. HwU National Cheng Kung University, R.O. China

B. L. KariHaloo University of Wales, U.K.

Y. Y. KIM Seoul National University, Republic of Korea

Z. Mroz Academy of Science, Poland

D. PAMPlonA Universidade Católica do Rio de Janeiro, Brazil

M. B. RUBIN Technion, Haifa, Israel

A. N. SHUPIKov Ukrainian Academy of Sciences, Ukraine

T. TARNAI University Budapest, Hungary

F. Y. M. WAN University of California, Irvine, U.S.A.

P. WRIGGERS Universität Hannover, Germany

W. YANG Tsinghua University, P.R. China

F. ZIEGLER Technische Universität Wien, Austria

\section{PRODUCTION}

\section{Paulo Ney de Souza Production Manager \\ SheIla Newbery Senior Production Editor \\ SILVIO LEVY Scientific Editor}

See inside back cover or http://www.jomms.org for submission guidelines.

JoMMS (ISSN 1559-3959) is published in 10 issues a year. The subscription price for 2010 is US $\$ 500 /$ year for the electronic version, and \$660/year (+\$60 shipping outside the US) for print and electronic. Subscriptions, requests for back issues, and changes of address should be sent to Mathematical Sciences Publishers, Department of Mathematics, University of California, Berkeley, CA 94720-3840.

JoMMS peer-review and production is managed by EditFLOW ${ }^{\mathrm{TM}}$ from Mathematical Sciences Publishers.

PUBLISHED BY

mathematical sciences publishers

http://www.mathscipub.org

A NON-PROFIT CORPORATION

Typeset in LATEX

CCopyright 2010. Journal of Mechanics of Materials and Structures. All rights reserved. 


\title{
Journal of Mechanics of Materials and Structures
}

\author{
Volume 5, No. 2 February 2010
}

A critical analysis of interface constitutive models for the simulation of delamination in composites and failure of adhesive bonds

Anton Matzenmiller, Sebastian Gerlach and Mark Fiolka

185

Computational studies of collagen fibril biominerals using a virtual internal bond

model with extrinsic length scale

Ganesh Thiagarajan and Kavita Deshmukh

The simulation of stochastically excited viscoelastic systems and their stability

VADIM D. POTAPOV

Fundamental solutions for an inhomogeneous cross-anisotropic material due to horizontal and vertical plane strain line loads

Cheng-Der Wang, Jia-YAN Hou and WeI-Jer WANG

Mechanical and fracture analysis of welded pearlitic rail steels

Aldinton Allie, Heshmat A. Aglan and Mahmood Fateh

Rate dependence of indentation size effects in filled silicone rubber

Ramanjaneyulu V. S. TATIRAJU and ChUng-SOUK HAN

A novel application of a laser Doppler vibrometer in a hèalth monitoring system

DAVOOD REZAEI and FARID TAHERI

Energy absorption of a helicoidal bistable structure

Seubpong Leelavanichkul, Andrej Cherkaev, Daniel O. Adams

and FLORIAN SOLZBACHER

Decay properties of solutions of a Mindlin-type plate model for rhombic systems

Francesca Passarella, Vincenzo Tibullo and VitTorio Zampoli

A consistent refinement of first-order shear deformation theory for laminated composite and sandwich plates using improved zigzag kinematics Alexander Tessler, Marco Di Sciuva and Marco Gherlone 